\title{
Latitudinal wave coupling of the stratosphere and mesosphere during the major stratospheric warming in 2003/2004
}

\author{
D. Pancheva ${ }^{1,2}$, P. Mukhtarov ${ }^{2}$, N. J. Mitchell ${ }^{1}$, B. Andonov ${ }^{2}$, E. Merzlyakov ${ }^{3}$, W. Singer ${ }^{4}$, Y. Murayama ${ }^{5}$, \\ S. Kawamura ${ }^{5}$, J. Xiong ${ }^{6}$, W. Wan ${ }^{6}$, W. Hocking ${ }^{7}$, D. Fritts ${ }^{8}$, D. Riggin ${ }^{8}$, C. Meek ${ }^{9}$, and A. Manson ${ }^{9}$ \\ ${ }^{1}$ CSAOS, Dept. of Electronic \& Electrical Engineering, University of Bath, Bath, UK \\ ${ }^{2}$ Geophysical Institute, Bulgarian Academy of Sciences, Sofia, Bulgaria \\ ${ }^{3}$ Institute of Experimental Meteorology, Obninsk, Russia \\ ${ }^{4}$ Leibniz-Institute of Atmospheric Physics, Kühlungsborn, Germany \\ ${ }^{5}$ National Institute of Information and Communications Technology, Tokyo, Japan \\ ${ }^{6}$ Institute of Geology and Geophysics, Beijing, China \\ ${ }^{7}$ University of Western Ontario, London, Canada \\ ${ }^{8}$ CoRA/North West Research Associates, Boulder, USA \\ ${ }^{9}$ ISAS, University of Saskatchewan, Saskatoon, Canada
}

Received: 6 September 2007 - Accepted: 9 January 2008 - Published: 26 March 2008

\begin{abstract}
The coupling of the dynamical regimes in the high- and low-latitude stratosphere and mesosphere during the major SSW in the Arctic winter of 2003/2004 has been studied. The UKMO zonal wind data were used to explore the latitudinal coupling in the stratosphere, while the coupling in the mesosphere was investigated by neutral wind measurements from eleven radars situated at high, highmiddle and tropical latitudes. It was found that the inverse relationship between the variability of the zonal mean flows at high- and low-latitude stratosphere related to the SSW is produced by global-scale zonally symmetric waves. Their origin and other main features have been investigated in detail. Similar latitudinal dynamical coupling has been found for the mesosphere as well. Indirect evidence for the presence of zonally symmetric waves in the mesosphere has been found.
\end{abstract}

Keywords. Meteorology and atmospheric dynamics (General circulation; Middle atmosphere dynamics; Waves and tides)

\section{Introduction}

The sudden stratospheric warming (SSW) is a violent largescale thermodynamical phenomenon in the winter polar region, which strongly affects the middle atmosphere, causing significant variations in the mesosphere-lower thermosphere (MLT) as well. It involves considerable changes of the back-

Correspondence to: D. Pancheva

(dpancheva@geophys.bas.bg) ground wind, temperature, planetary and gravity wave activity and redistributes ozone and other chemicals at high latitudes.

The key mechanism behind the SSW, initially proposed by Matsuno (1971) and now widely accepted, relates to the growth of upward propagating transient planetary waves and their non-linear interaction with the zonal mean flow. The interaction decelerates and/or reverses the eastward winter winds in the stratosphere-mesosphere system and also induces a downward circulation in the stratosphere causing adiabatic heating and an upward circulation in the mesosphere resulting in adiabatic cooling (Liu and Roble, 2002, 2005; Coy et al., 2005). These basic features of the stratospheremesosphere manifestation of the SSW have been supported by many observations (Lysenko et al., 1975; Gregory and Manson, 1975; Myrabø et al., 1984; Cevolani, 1989; Manson and Meek, 1991; Singer et al., 1992; Whiteway and Carswell, 1994; Hoffmann et al., 2002, 2007; Jacobi et al., 2003; Dowdy et al., 2004; Walterscheid et al., 2000; Hernandez, 2003; Cho et al., 2004; Siskind et al., 2005; Mukhtarov et al., 2007).

While the effects of the SSW on the high-latitude stratosphere-mesosphere system have been well researched, there are only a few reports devoted to the tropical and lowlatitude middle atmosphere response to the SSW. Julian and Labitzke (1965) were the first to note that the influence of the Northern Hemisphere ( $\mathrm{NH}$ ) warming can be seen in the subtropical atmosphere as well. The authors found two circulation cells, polar and subtropical, which intensify during the SSW. An association between the high-latitude stratosphere warming and the tropical and even equatorial summer

Published by Copernicus Publications on behalf of the European Geosciences Union. 
hemisphere cooling was revealed by the Nimbus III satellite data (Fritz and Soules, 1970). The out-of-phase relationship in the stratospheric temperature at high- and low-latitudes was evident only in the radiances when averaged around latitude circles, i.e. in zonal mean temperature. Mukhtarov et al. (2007) using the UK Met. Office (UKMO) data investigated the latitudinal structure of the genuine stratospheric anomalies (emphasized by removing the UKMO climatology) related to the major SSW in the Arctic winter of 2003/2004 and found that: (i) warmings at high latitudes are associated with coolings at low-middle and tropical latitudes, and (ii) negative zonal mean zonal wind anomalies (deceleration/reversal of the eastward winter flow) at high latitudes are linked with the positive (amplification of the eastward flow) anomalies at low-middle and tropical latitudes; these effects are particularly strong in the upper stratosphere. An extensive study of the low-latitude $\left(5-15^{\circ} \mathrm{N}\right)$ stratospheremesosphere response to a few SSW has been recently reported by Shepherd et al. (2007), where the authors use ground based and satellite data in order to understand better the global effects of the SSW.

The present work is focused on the latitudinal coupling observed in the stratosphere and mesosphere during the major SSW in the Arctic winter of 2003/2004. A major SSW took place in December/January which was particularly remarkable because it consisted of a period of nearly two months of polar vortex disruption and a reversed direction of the zonal mean flow observed in the middle and lower stratosphere (Manney et al., 2005). The purpose of the study is to examine the type of planetary waves which produce the latitudinal relationship between the dynamical regimes of high- and tropical latitudes (Mukhtarov et al., 2007) and to clarify their forcing mechanism. The UK Met Office zonal wind data have been used to explore the latitudinal structure of the anomalies in the NH stratosphere. The mesospheric anomalies related to the major SSW in 2003/2004 have been identified in the neutral wind measurements made by eleven radars situated at high, high-middle and tropical latitudes. The latitudinal relationship between the temperature anomalies related to this major SSW will be investigated in a separate work where the SABER temperature data will be used as well.

\section{Observations and data analysis}

\subsection{UK Met Office data}

The UKMO data set is used to examine the features of the global planetary waves present in the winter of 2003/2004 stratosphere of the Northern Hemisphere (NH). This data set is a result of assimilation of in situ and remotely sensed data into a numerical analysis model of the stratosphere and troposphere. A description of the original data assimilation system can be found in Swinbank and O'Neill (1994); the new 3-D-variational system is described in Swinbank and Ort- land (2003). The outputs of the assimilation are global fields of daily temperature, geopotential heights and wind components at pressure levels from the surface up to $0.1 \mathrm{hPa}$. The daily data fields have global coverage with $2.5^{\circ}$ and $3.75^{\circ}$ steps in latitude and longitude, respectively. The UKMO data well represent the global features of stratospheric dynamics. The analysis in this paper focuses on studying in detail that type of planetary waves in the zonal wind which produce the latitudinal relationship observed in the NH stratosphere during the Arctic winter of 2003/2004.

The UKMO zonal data at four pressure levels (30, 10, 1 and $0.3 \mathrm{hPa}$ ) are used to explore the features of the global planetary waves in the $\mathrm{NH}$ stratosphere in the period of time 1 October 2003-30 April 2004. Since the occurrence of SSW is related to the growth of planetary waves in the winter stratosphere, we investigate both the stationary Rossby planetary waves (SPWs) that propagate upward from the troposphere and the travelling planetary waves that are also present in the UKMO data. To determine the predominant periods of the planetary waves we use a spectral analysis method that is a two-dimensional analogue of the Lomb-Scargle periodogram method (Lomb, 1975; Scargle, 1982) based on a least-squares fitting procedure applied to the entire time series. The planetary waves with periods between 5 and 30 days and with zonal wavenumbers up to 3 are studied. The main purpose is not only to define the predominant periods of the wave components that contribute to the variability of the atmospheric fields but also to isolate and to study them in detail. To extract the waves from the data and to determine their amplitudes and phases, we use again a least-squares fitting procedure, but this time it is applied to the time segment twice the length of the longest period under investigation. Then this segment is moved through the time series with steps of 1 day in order to obtain daily values of the wave amplitudes and phases. The planetary wave structures (SPWs and travelling planetary waves together) are viewed in the zonal wind data for the four pressure levels.

\subsection{MLT neutral wind measurements}

We use zonal wind data from 4 radars situated at high latitudes between $63^{\circ} \mathrm{N}$ and $69^{\circ} \mathrm{N}, 4$ radars at high-middle latitudes between $52^{\circ} \mathrm{N}$ and $55^{\circ} \mathrm{N}$ and 3 radars at tropical latitudes between $21^{\circ} \mathrm{N}$ and $31^{\circ} \mathrm{N}$ (Table 1). Some details about the radars at high- and high-middle latitudes and their routine data analysis procedures can be found in Mukhtarov et al. (2007). The radar at Wuhan is commercially produced ATRAD meteor radar (Xiong et al., 2004), while the radars at Yamagawa and Kauai are medium frequency (MF) radars. A detailed description of both radars can be found in $\mathrm{Mu}-$ rayama et al. (2000) and Riggin et al. (2004).

The basic information used in this study consists of daily estimates of the prevailing zonal wind at each of the height intervals. The daily zonal winds are obtained by applying a linear least-squares fitting algorithm to time segments of 
Table 1. Geographic locations of the radars, the type of the instrument and the height range of available measurements.

\begin{tabular}{lccc}
\hline Station & Instrument & Location & Height range $(\mathrm{km})$ \\
\hline Andenes & SKiYMET & $69^{\circ} \mathrm{N}, 16^{\circ} \mathrm{E}$ & $82-98$ \\
Esrange & SKiYMET & $68^{\circ} \mathrm{N}, 21^{\circ} \mathrm{E}$ & $81-97$ \\
Poker Flat & MF radar & $65^{\circ} \mathrm{N}, 147^{\circ} \mathrm{W}$ & $50-108$ \\
Yellowknife & SKiYMET & $63^{\circ} \mathrm{N}, 114^{\circ} \mathrm{W}$ & $82-98$ \\
Obninsk & Meteor radar & $55^{\circ} \mathrm{N}, 37^{\circ} \mathrm{E}$ & No height resolution \\
Kuehlungsborn & MST (meteor mode) & $54^{\circ} \mathrm{N}, 12^{\circ} \mathrm{E}$ & 85 and 94 \\
Castle Eaton & Meteor radar & $52^{\circ} \mathrm{N}, 2^{\circ} \mathrm{W}$ & No height resolution \\
Saskatoon & MF radar & $52^{\circ} \mathrm{N}, 107^{\circ} \mathrm{W}$ & $67-94$ \\
Yamagawa & MF radar & $31^{\circ} \mathrm{N}, 131^{\circ} \mathrm{E}$ & $50-108$ \\
Wuhan & ATRAD & $30.5^{\circ} \mathrm{N}, 114^{\circ} \mathrm{E}$ & $70-108$ \\
Kauai & MF radar & $21^{\circ} \mathrm{N}, 159.6^{\circ} \mathrm{W}$ & $60-98$ \\
\hline
\end{tabular}

1-day duration; a superposition of mean wind and 24-, 12and 8-h harmonic components was fitted. The segment was then incremented through the zonal wind time series in steps of one day in order to yield daily-spaced values of the prevailing wind. The prevailing zonal wind and tidal characteristics are calculated at each step only if at least 16 valid data are present in the 1-day time segment. The daily values of the zonal winds are used later for studying the planetary waves observed in the MLT region.

The planetary waves are transient phenomena and they are studied by a wavelet transform method. The wavelet analysis presented here employs the continuous Morlet wavelet, which consists of a plane wave modulated by a Gaussian envelope. The non-dimensional frequency, which gives the number of oscillations within the wavelet itself, is set to six to satisfy the wavelet admissibility condition (Torrence and Compo, 1998). The Morlet wavelet was selected because of its simplicity and convenience in investigating wave-like events observed in the neutral wind of the MLT region (Pancheva and Mukhtarov, 2000). The localization characteristics in time and frequency space of the Morlet wavelet used in this study are as follows: the time localization, or the so called "cone of influence", is defined as a time interval which contributes to the wavelet coefficient at a given instant $t_{o}$. In our case the influence cone is: ,$t \in\left[t_{0}-\sqrt{2} a, t_{0}+\sqrt{2} a\right]$ where $a$ is the wavelet scale and $1.03 a=T$, where $T$ is the Fourier period. Likewise, the localization characteristics of the wavelet in frequency space give local information about the function in frequency range: $\omega \in\left[\frac{\omega_{0}}{a}-\frac{1}{a \sqrt{2}} ; \frac{\omega_{0}}{a}+\frac{1}{a \sqrt{2}}\right]$.

It is worth noting that in the MLT region we cannot divide the planetary waves found in the radar measurements into SPWs and travelling planetary waves. The reason is that the number of the radars is far from being sufficient for applying a 2-dimensional (time-longitude) analogue of the LombScargle periodogram method used with the UKMO data. As a result of this some disturbances found in the MLT zonal

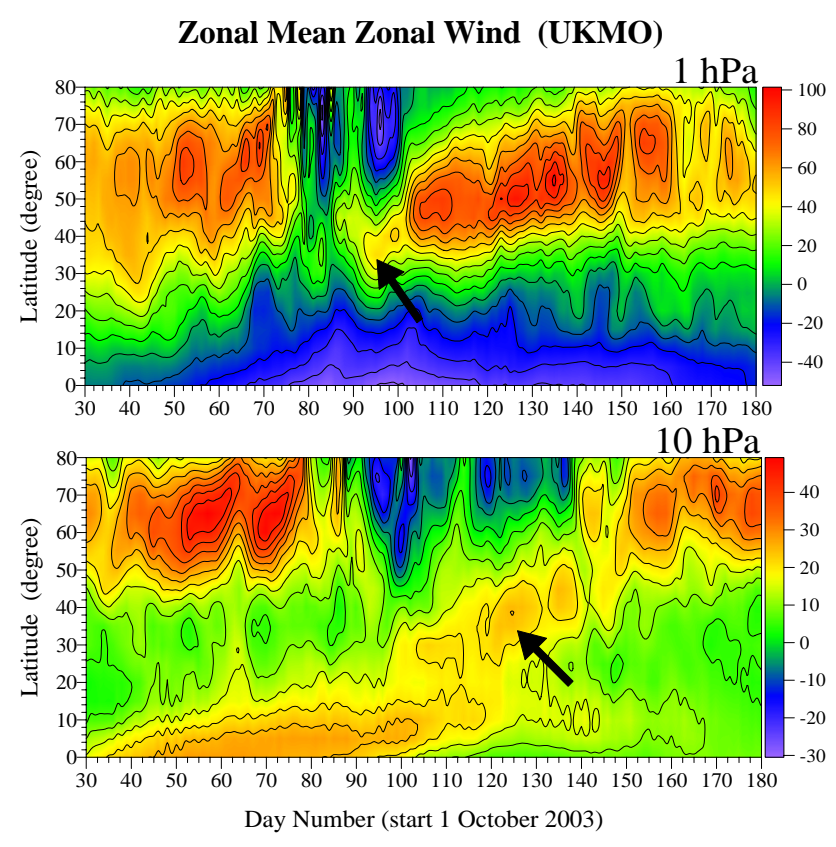

Fig. 1. Height-time cross sections of the zonal mean zonal wind in the stratosphere at pressure levels 10 and $1 \mathrm{hPa}$ during the period of time between November 2003 and March 2004.

wind could be affected by a perturbation with similar period but with different zonal structure.

\section{Latitudinal wave coupling in the stratosphere}

Figure 1 shows the height-time cross section of the zonal mean zonal wind in the stratosphere at pressure levels 10 and $1 \mathrm{hPa}$ during the period of time between November 2003 and March 2004. The middle stratosphere $(10 \mathrm{hPa}, \sim 32 \mathrm{~km}$ height) polar vortex disruption related to the major SSW (that began in late December, near day number 90) is well outlined between late December and February (between day 


\section{Spectra of Travelling Planetary Waves (zonal wind)}
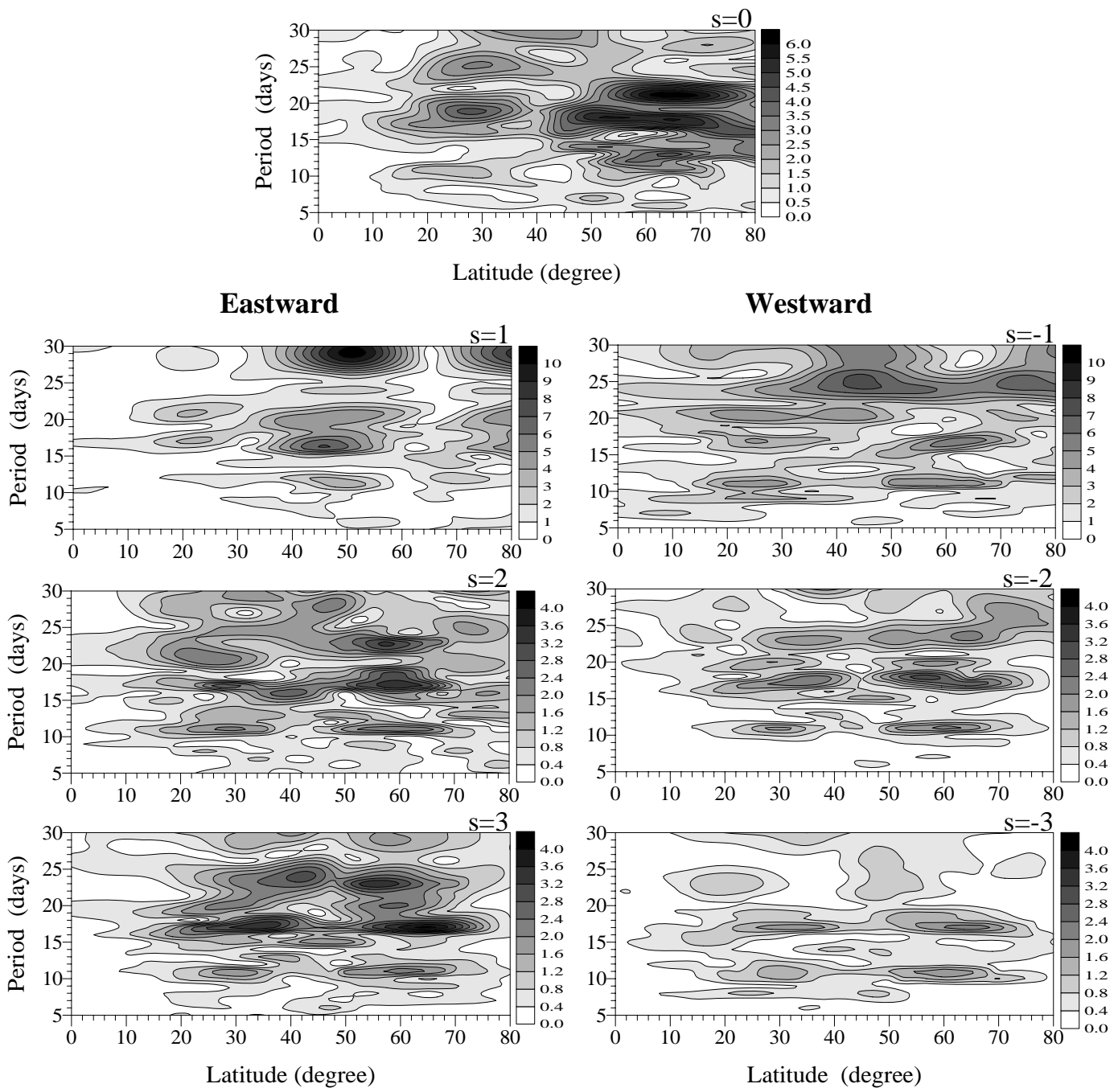

Fig. 2. Latitudinal amplitude spectra of the zonally symmetric ( $s=0)$ waves (upper plot), eastward (left column of plots) and westward (right column of plots) travelling waves with zonal wavenumber up to 3 calculated from the zonal wind data at $1 \mathrm{hPa}$ pressure level for the time interval of 1 October 2003-30 April 2004.

numbers 90-140) at high-middle and high latitudes and it is associated with strengthening of the eastward zonal mean zonal wind at lower latitudes (it is marked by a tick arrow between $\left.20-40^{\circ} \mathrm{N}\right)$. The upper stratosphere $(1 \mathrm{hPa}, \sim 48 \mathrm{~km})$ polar vortex broke up earlier than that at $10 \mathrm{hPa}$, at middle of December (about day number 75), but began to recover by early January (about day number 100) and it strengthened significantly in late January-March. An inverse relationship between the zonal mean flow deceleration/reversal at high latitudes and the strengthening of the eastward zonal mean flow at tropical latitudes is again visible in the upper plot of Fig. 1 (marked by a tick arrow), but only between December and early January (about day numbers 85-100).
It is important to note that the inverse relationship between the dynamical regimes at high and tropical latitudes is observed not only between the background zonal mean flows at both latitude ranges, but also between the perturbations with periods of planetary waves (2-30 days) present in the zonal mean zonal wind. Figure 1 shows that not only the high-latitude negative (deceleration/reversal of the eastward winter flow) perturbations are linked with positive (amplification of the eastward flow) ones at tropical latitudes during the major SSW event, but later the positive wind perturbations observed between $50-60^{\circ} \mathrm{N}$ are associated with the negatives anomalies at low-latitudes; the latter are particularly visible at the upper plot of Fig. 1 after the strengthening of the polar vortex in the upper stratosphere. The presence 


\section{6-Day (s=0) Wave (zonal wind)}

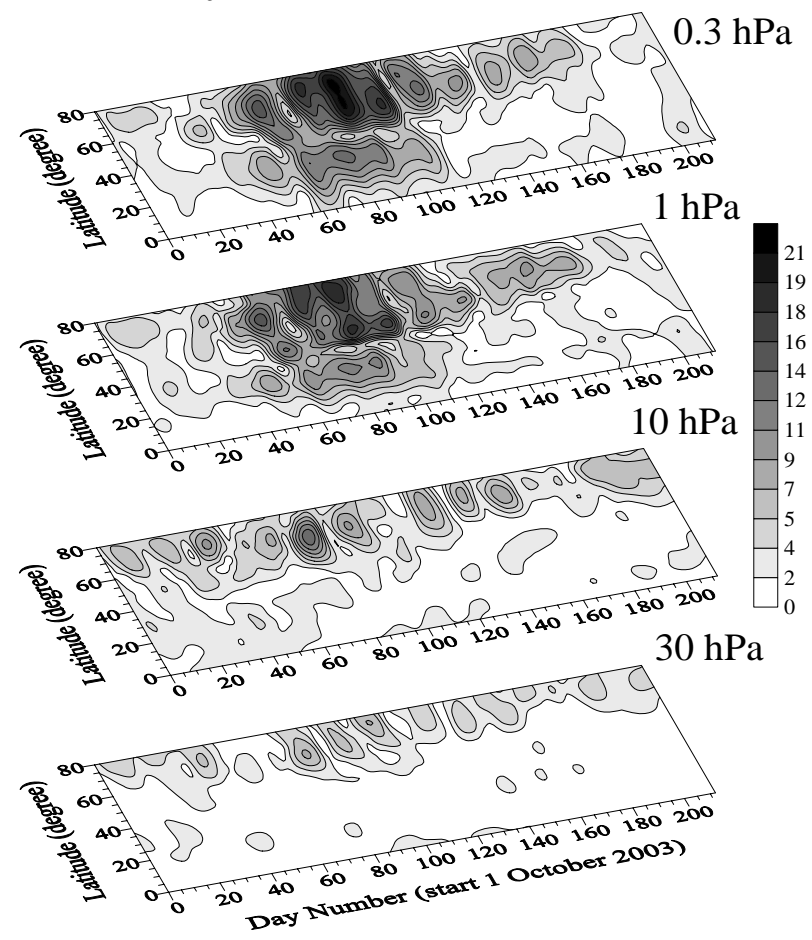

\section{1-Day (s=0) Wave (zonal wind)}

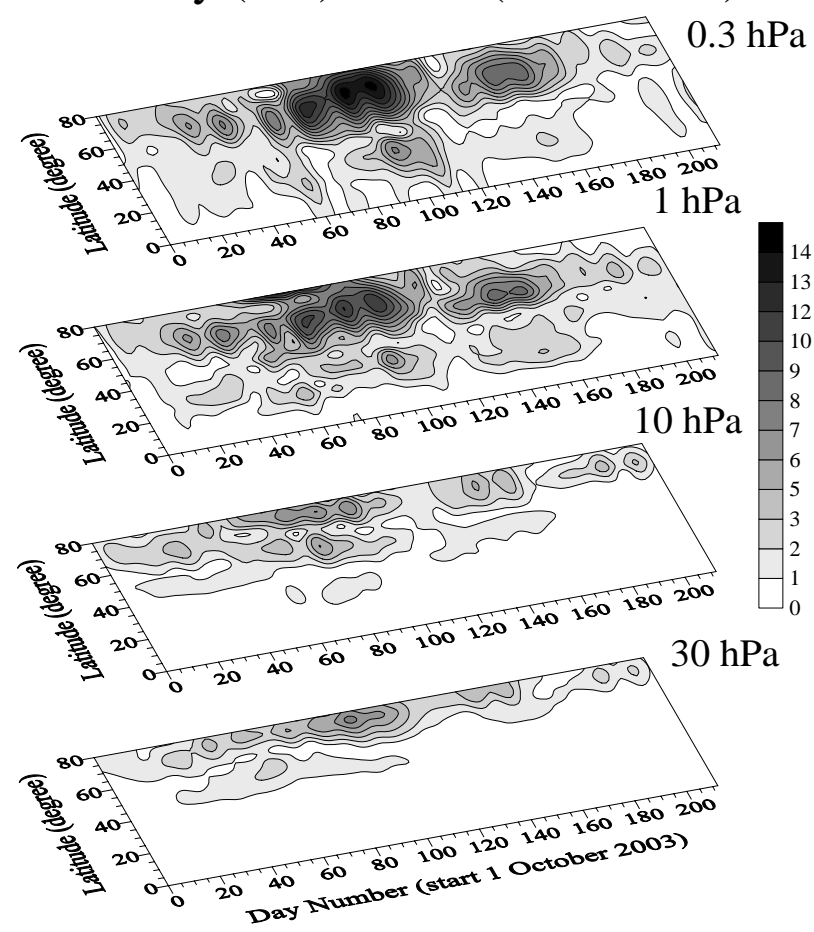

Fig. 3. Latitude-time cross sections of the amplitudes of $\sim 16$ - (left column of plots) and $\sim 11$-day (right column of plots) zonally symmetric waves at pressure levels: $0.3,1,10$ and $30 \mathrm{hPa}$; the latitude range of $0-80^{\circ} \mathrm{N}$ is studied.

of the planetary perturbations in the zonal mean zonal wind (the zonal wind averaged across longitude) means that these waves do not depend on the longitude, or in other words their zonal wavenumber should be zero.

\subsection{Main features of zonally symmetric waves}

Pancheva et al. (2007) presented evidence of global-scale zonally symmetric $(s=0)$ waves with large amplitudes observed in the winter of 2003/2004. These waves which play an important role in coupling the dynamical regimes of the high- and low-latitude stratosphere and their forcing mechanism will be studied in detail here. For this purpose we need first to determine their periods and then to isolate them from the data in order to study their main features. It is well known that the time period preceding the onset of the SSW is usually characterized by more than one type of planetary waves present concurrently in the stratosphere. Among them are the quasi-stationary planetary waves (SPWs) which are the strongest waves in the winter stratosphere, and the zonally travelling waves, usually related to the normal modes of the atmosphere. To avoid the distortion of the zonally symmetric waves in isolating them from the data, we need to extract simultaneously all significant waves present in the data. Therefore, we need to study all planetary waves observed in the winter stratosphere of 2003/2004.
To determine the wave periods we use a two-dimensional analogue of the Lomb-Scargle periodogram method (Lomb, 1975; Scargle, 1982) based on a least-squares fitting procedure applied to the entire time series (1 October 2003-30 April 2004). The planetary waves with periods between 5 and 30 days and zonal wavenumbers up to 3 are studied here. The upper plot of Fig. 2 shows the latitudinal amplitude spectrum of zonally symmetric $(s=0)$ waves, while the left and right columns of plots - the spectra of, respectively, eastward and westward travelling waves in the zonal wind at $1 \mathrm{hPa}$ pressure level. The spectrum of zonally symmetric waves indicates 3 prevailing periods: $\sim 22-24,15-17$ and 11-12 days. There are two latitudinal amplifications of these periods in the spectrum: the main amplification is centred around $50-60^{\circ} \mathrm{N}$ and the secondary one - around $20-40^{\circ} \mathrm{N}$. The spectral analysis revealed also that similar periods can be distinguished in the spectra of eastward and westward travelling wave at all zonal wavenumbers. This fact is an indication for a possible nonlinear interaction between the SPW and the travelling wave whereby new travelling waves are generated with the same period as that of the primary travelling wave, but with different zonal wavenumbers. This feature of the zonal wind spectra will be used later in defining the forcing mechanism of the zonally symmetric $(s=0)$ waves.

To extract the waves from the data or to determine their amplitudes and phases, we use again a least-squares fitting 

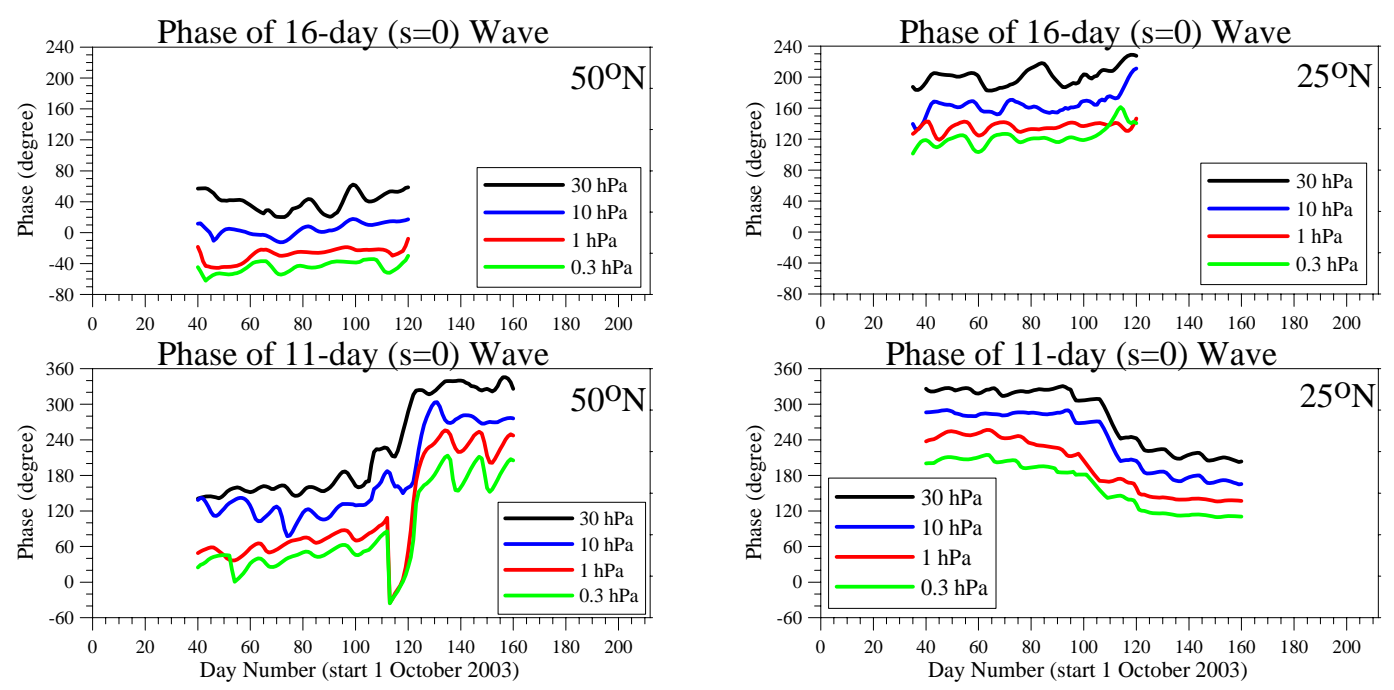

Fig. 4. Phases of the $\sim 16$ - (upper row of plots) and $\sim 11$-day (bottom row of plots) zonally symmetric waves at $50^{\circ} \mathrm{N}$ (left column of plots) and $25^{\circ} \mathrm{N}$ (right column of plots); the phases are presented at studied four pressure levels.

\section{SPW1 in Zonal Wind}

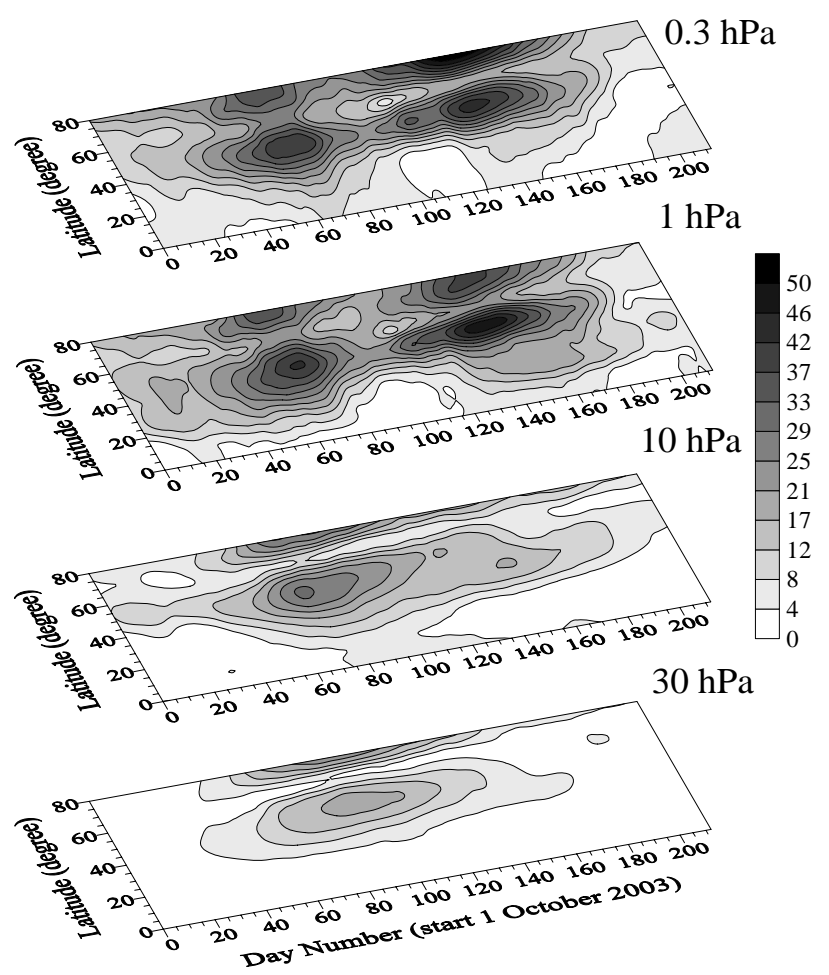

SPW2 in Zonal Wind

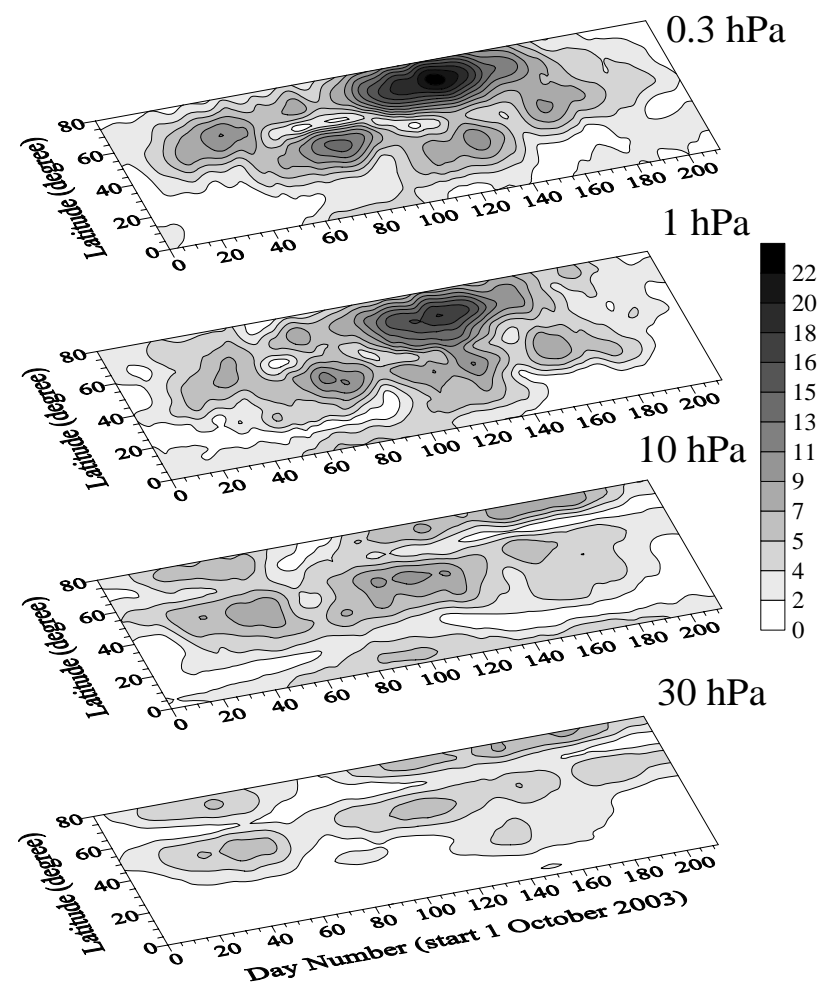

Fig. 5. Latitude-time cross sections of the SPW1 (left column) and SPW2 amplitudes (right column) in the UKMO zonal wind data at four pressure levels: $30,10,1$, and $0.3 \mathrm{hPa}$.

procedure, but this time it is applied to a time segment twice the length of the longest period under investigation. Then this segment is moved through the time series with steps of 1 day in order to obtain daily values of the wave amplitudes and phases. The decomposition includes: (i) the first three modes of the SPWs (i.e. SPWs with zonal wavenumbers 1, 2 and 3), (ii) the travelling (eastward and westward) waves with periods of $\sim 22-23,16$ and 11 days and zonal wavenumbers up 


\section{SPW1 in Zonal Wind}
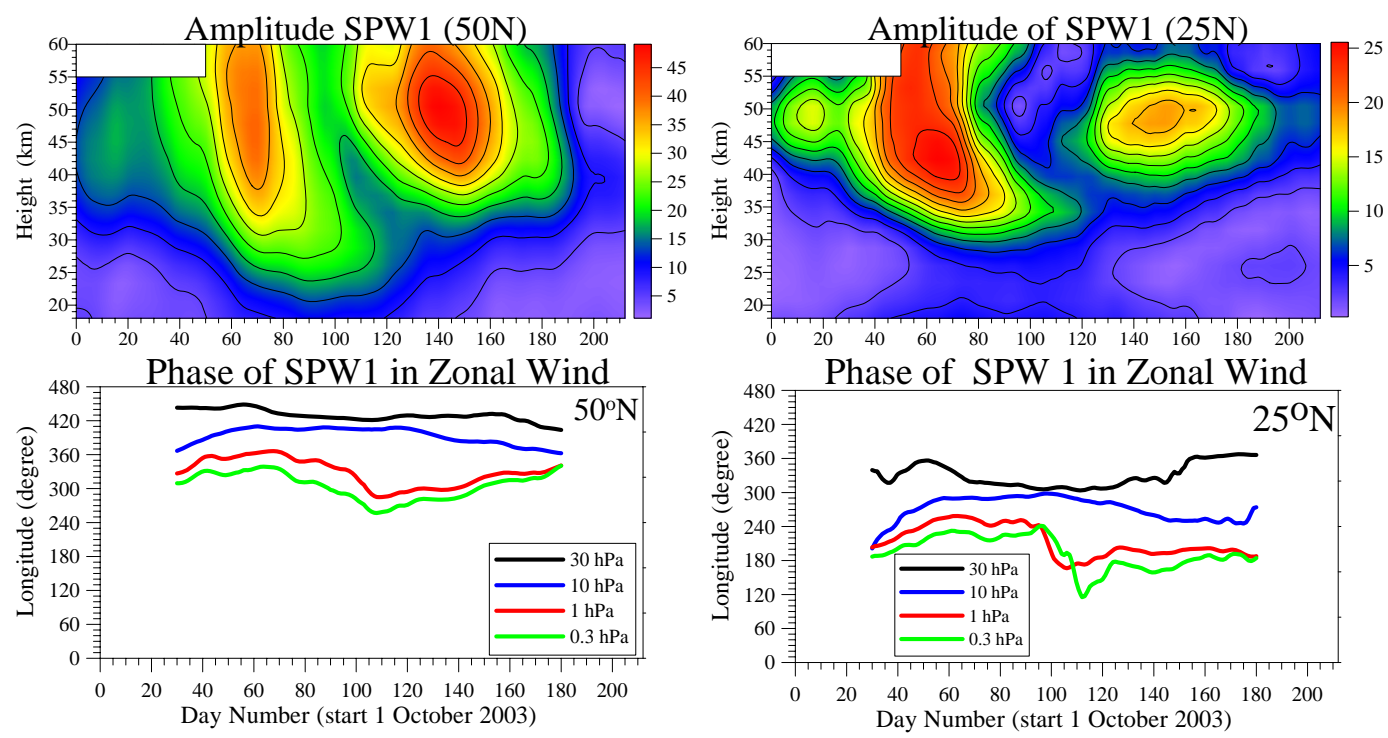

Fig. 6. Height-time cross sections of the SPW1 amplitudes (upper row of plots) and their phases (bottom row of plot) at latitudes of $50^{\circ} \mathrm{N}$ (left column of plots) and $25^{\circ} \mathrm{N}$ (right column of plots).

to 3 , and (iii) the zonally symmetric waves with the same periods as those of the travelling waves. All oscillations are extracted simultaneously in order to avoid a possible distortion of the usually weaker travelling waves by the stronger SPWs. The length of the time segment used for performing the least-squares fitting procedure is 45 days. We note that in order to cover the entire interval of time between 1 October 2003 and 30 April 2004 with daily values of the wave parameters we expand this interval by adding 22 days to each side (9 September 2003-22 May 2004).

Figure 3 shows the latitude-time cross sections of the amplitudes of $\sim 16$ - and $\sim 11$-day zonally symmetric waves at the considered four pressure levels; the latitude range of $0-$ $80^{\circ} \mathrm{N}$ is studied (we note that the $\sim 23$-day $(s=0$ ) wave will not be considered here as it defines some isolated very strong disturbances present at high and low latitude stratosphere). Both patterns are very similar and show first, that there are two branches of amplifications centred at $50-60^{\circ} \mathrm{N}$ and $20-$ $30^{\circ} \mathrm{N}$ and second, that the main amplification is between day number 60 and 100 (late November/early January) and the secondary one - between 120 and 160 (February-March). The main tropical amplification is particularly strong in the amplitudes of the 16-day zonally symmetric wave, while the secondary tropical amplification is present only in the 11-day $(s=0)$ wave. Both wave amplitudes grow significantly with height.

Figure 4 shows the phases of the $\sim 16$ - (upper row of plots) and $\sim 11$-day (bottom row of plots) zonally symmetric waves at $50^{\circ} \mathrm{N}$ (left column of plots) and $25^{\circ} \mathrm{N}$ (right column of plots) and at the four pressure levels. The phase distribu- tion with height indicates that the zonally symmetric waves are vertically upward propagating waves. To facilitate the phase comparison at high and low latitudes the scales are the same for all plots. For both periods the waves at high and low latitudes are almost out-of-phase (the phase difference is $\left.150-160^{\circ}\right)$. This feature of the zonally symmetric waves defines the opposite relationship between the perturbations in the dynamical regimes at high and low latitudes.

\subsection{Forcing mechanism of zonally symmetric waves}

It is known that there are no zonally symmetric normal modes with such long periods as those of the waves found in the UKMO zonal wind data (Longuet-Higgins, 1968). As we have already mentioned the fact that we found zonally symmetric and zonally travelling waves with similar periods present concurrently in the UKMO zonal wind data (Fig. 2) suggests that a possible forcing mechanism of the zonally symmetric waves could be a nonlinear interaction of the SPWs with the zonally propagating planetary waves. In this way the zonally symmetric waves with the periods of the zonally travelling waves should be produced. To describe how this coupling process works we note the following: if a given oscillation composed of two primary travelling and stationary waves defined by their frequency-wave number pairs $\left(\omega, s_{1}\right)$ and $\left(0, s_{2}\right)$ passes through a quadratic system, then the output from this system would yield the original primary waves plus four other secondary waves which have the following frequency-wave number pairs: $\left(2 \omega, 2 s_{1}\right),\left(0,2 s_{2}\right),(\omega$, $\left.s_{1}+s_{2}\right)$ and $\left(\omega, s_{1}-s_{2}\right)$ (Angelats i Coll and Forbes, 2002). 

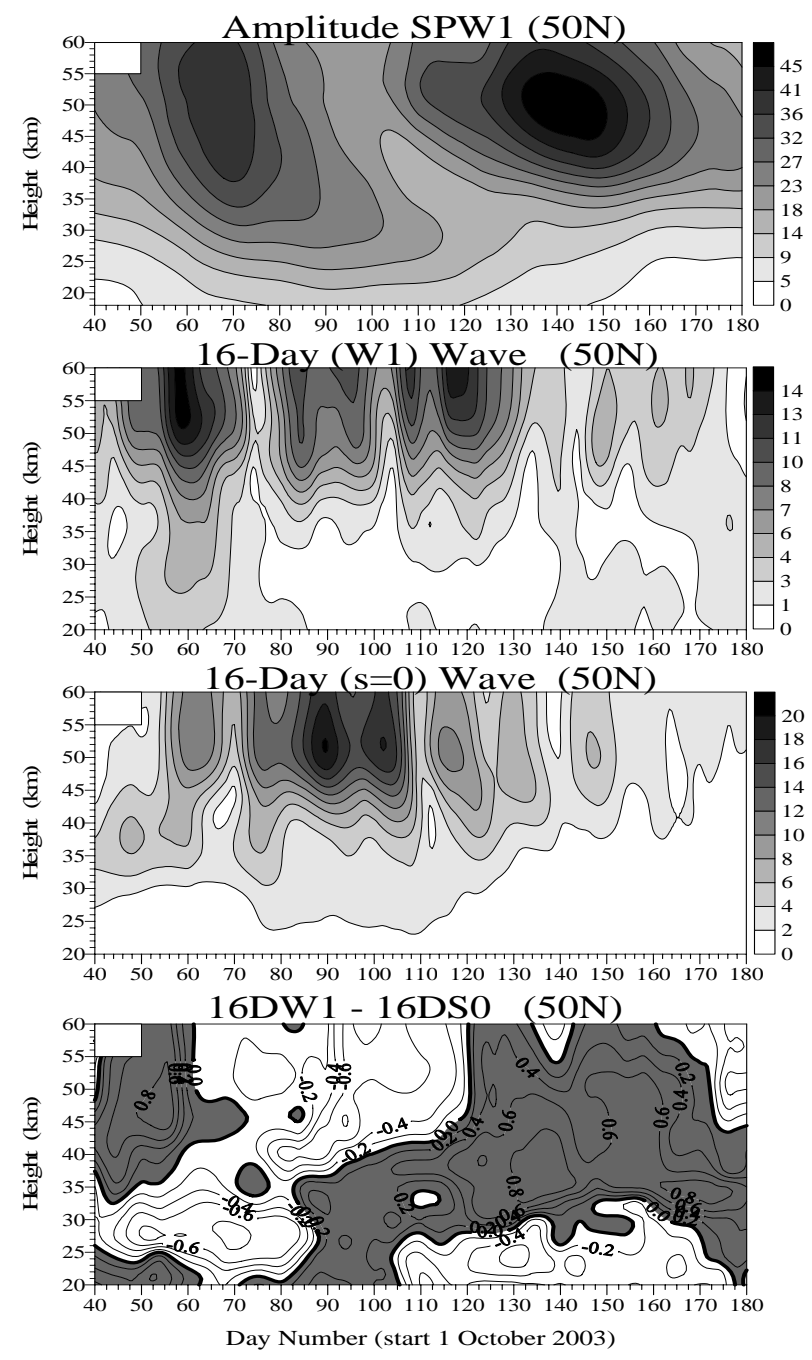
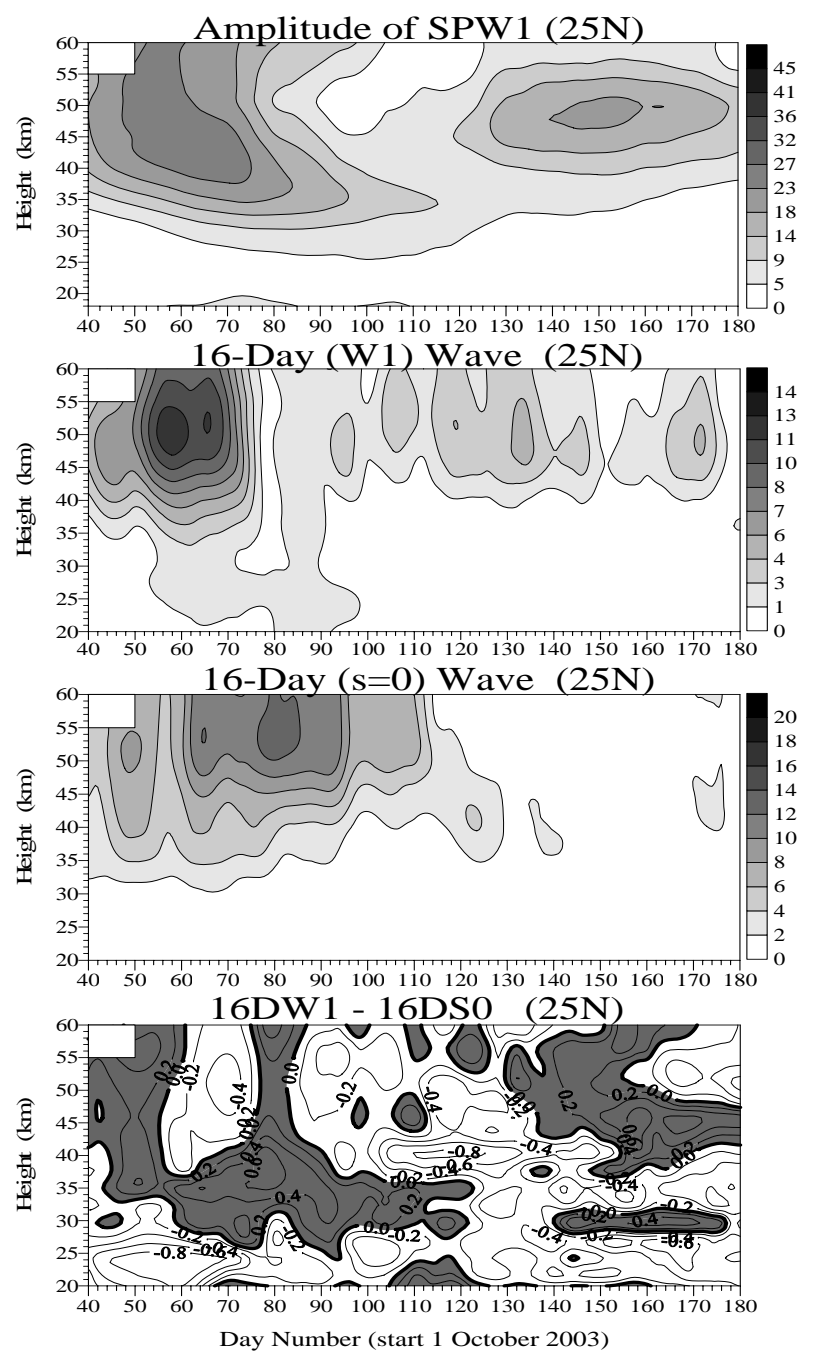

Fig. 7. Height-time cross sections of the amplitudes of: the primary SPW1 (upper row of plots), the 16-day W1 primary wave (second row of plots) and the 16-day ( $s=0)$ secondary wave (third row of plots) at $50^{\circ} \mathrm{N}$ (left column of plots) and $25^{\circ} \mathrm{N}$ (right column of plots) for the period of time November 2003-March 2004. The bottom row of plots shows height-time cross sections of the cross-correlation coefficients between the amplitudes of the 16-day W1 wave and the 16-day $(s=0)$ secondary wave at $50^{\circ} \mathrm{N}$ and $25^{\circ} \mathrm{N}$.

In this case the last two types of secondary waves are of interest to us. They are known as "sum" and "difference" secondary waves. Through such a process the presence of zonally symmetric wave with frequency $\omega$ and wave number $s=0$ could be due to the interactions of: (i) the SPW1 with eastward or with westward travelling planetary wave with frequency $\omega$ and zonal wavenumber 1 ; in the first case the zonally symmetric wave will be the "sum" secondary wave, while in the second one - the "difference" secondary wave, and (ii) the SPW2 with eastward/westward travelling planetary wave with frequency $\omega$ and zonal wavenumber 2 ; similarly, in the first case the zonally symmetric wave would be the "sum"/"difference" secondary wave. Usually the amplitude of the SPW3 is significantly smaller than those of the waves 1 and 2 (in this case as well) and its contribution to the forcing is negligible. Figure 2 clearly indicated the presence of eastward and westward propagating waves with zonal wave numbers 1 and 2 and with periods $\sim 16$ and $\sim 11$ days, therefore the zonally symmetric waves could be produced by some of the above mentioned coupling processes.

It is worth mentioning that when the SPW1 interacts with the eastward/westward travelling wave with zonal wave number 1 , in addition to the zonally symmetric wave, the coupling also produces eastward/westward propagating wave with zonal wavenumber 2 . Therefore, the interaction between the stationary and travelling planetary waves also generates travelling waves with the same period but with higher than the primary wave zonal wave number. Apparently, during the winter of 2003/2004 the wave-wave coupling processes must have been quite strong in order to 

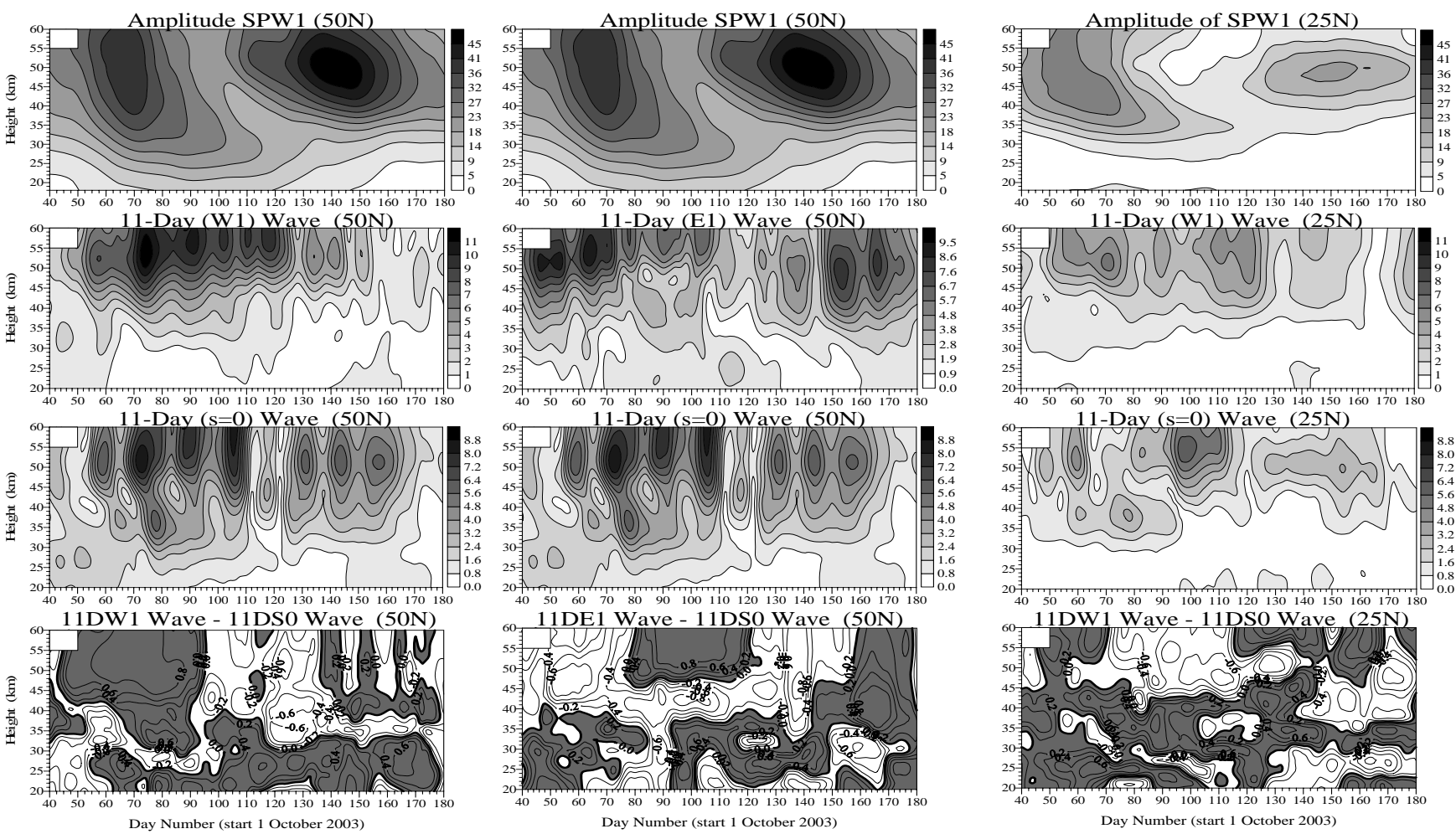

Fig. 8. The same as Fig. 7 but for the $\sim 11$-day W1 and $\sim 11$-day E1 primary waves and $\sim 11$-day $(s=0)$ secondary wave.

produce travelling waves with similar periods having zonal wave numbers up to 3 (note that only wave numbers up to 3 have been studied in this paper; see Fig. 2).

To clarify which SPW has dominant contribution to the generation of the zonally symmetric waves we extract both waves from the UKMO data in order to compare their pattern with that of the zonally symmetric waves. Figure 5 shows the latitude-time cross sections of the SPW1 (left column) and SPW2 amplitudes (right column) in the UKMO zonal wind data at four pressure levels: $30,10,1$, and $0.3 \mathrm{hPa}$. The zonal wind maxima of SPW1 are centred near $40-50^{\circ} \mathrm{N}$ and the North Pole. It shows two amplifications centred near days 60-70 and 130-140, a pattern very similar to that of the zonally symmetric waves (Fig. 2). The second amplification is seen mainly in the upper stratosphere (above $10 \mathrm{hPa}$ pressure level) similarly to the $\sim 11$-day $(s=0)$ wave which is observed only in the upper stratosphere between days 120-160. The SPW2 amplifies around and after the maximum of the SSW, near days $100-140$, in the high latitude upper stratosphere; its pattern is quite different from that of the zonally symmetric waves (Fig. 2).

From the comparison of Figs. 2 and 5 it follows that the SPW1 has dominant contribution to the generation of the zonally symmetric waves. Of course, the contribution of the SPW2 cannot be ignored, but if it participates in the forcing its effect is of secondary importance.
The next step of our analysis is related to a detailed study of the primary waves participating in the forcing of the $\sim 16$ and $\sim 11$-day $(s=0)$ waves. The wave characteristics at $50^{\circ} \mathrm{N}$ and $25^{\circ} \mathrm{N}$ will be considered. Figure 6 shows the height-time cross sections of the SPW1 amplitudes (upper row of plots) and phases (bottom row of plot) at both latitudes. The amplitudes at both latitudes have similar pattern, but different maximum values; those at $50^{\circ} \mathrm{N}$ reach $\sim 50 \mathrm{~m} / \mathrm{s}$, while at $25^{\circ} \mathrm{N}$ reach $\sim 25 \mathrm{~m} / \mathrm{s}$. The first amplification of the SPW1 (centred near days 60-80) at $25^{\circ} \mathrm{N}$ is significantly stronger than the second one. This feature probably causes the main amplification of the zonally symmetric waves between day number 60 and 100 in the tropical stratosphere. Similarly to the zonally symmetric waves the phases of the SPW1 at four pressure levels are shown in the figure. The westward phase tilt with height indicates that the SPW1 at both latitudes are vertically upward propagating waves. However, there is a significant phase difference, $\sim 120-140^{\circ}$, between the SPW1 at high and low latitudes. Therefore, the observed phase difference between the zonally symmetric waves at high and low latitudes is partly due to the phase difference of the SPW1 at both latitudes.

The second primary wave taking place in the forcing of the 16-day $(s=0)$ wave should be the eastward or westward 16-day travelling wave. The phase analysis of both 16-day waves indicated that only the wave travelling westward is a vertically upward propagating wave. Therefore, only it could 


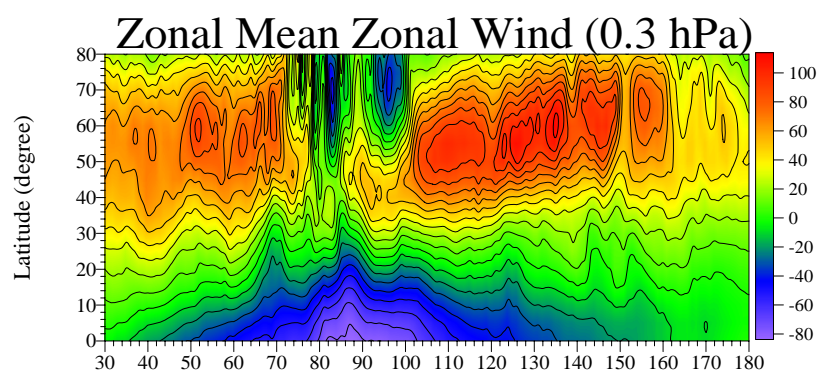

MLT Mean Zonal Wind
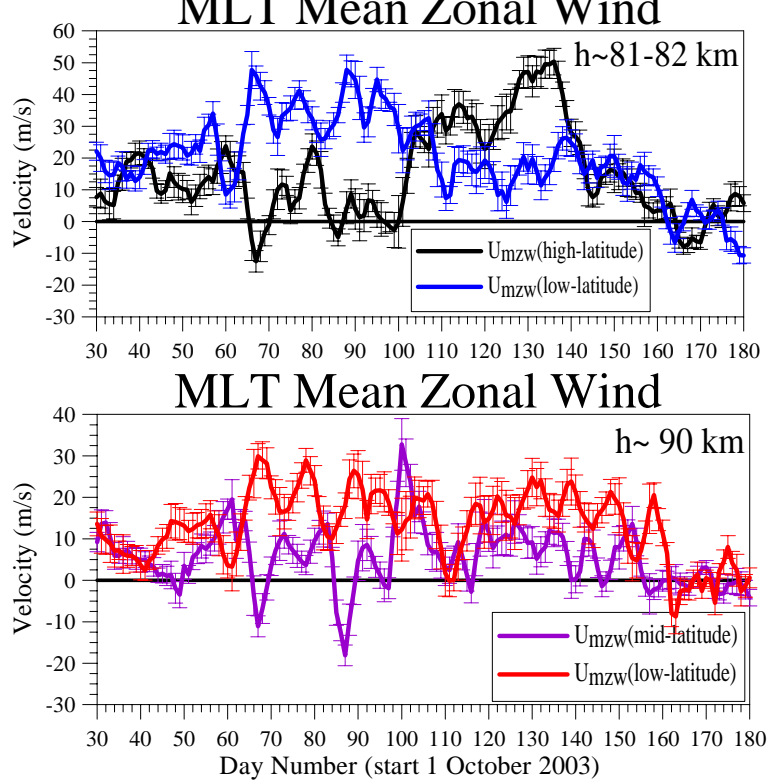

Fig. 9. Height-time cross section of the zonal mean zonal wind at pressure level $0.3 \mathrm{hPa}$ during the period of time between November 2003 and March 2004 (upper plot). The middle plot shows a comparison between the calculated mean zonal winds at $\sim 81-82 \mathrm{~km}$ for high (black) and tropical (blue) latitudes, while the bottom plot a comparison between the mean zonal winds at $\sim 90 \mathrm{~km}$ height for high-middle (purple) and tropical (red) latitudes (the error bars indicate the standard deviation of the mean).

contribute to the forcing of the 16-day $(s=0)$ wave. Figure 7 presents the time-height cross sections of the amplitudes of: the primary SPW1 (upper row of plots), the 16-day W1 primary wave (second row from above of plots) and the 16-day $(s=0)$ secondary wave (third row of plots) at $50^{\circ} \mathrm{N}$ (left column of plots) and $25^{\circ} \mathrm{N}$ (right column of plots) for the period of time November-March. An inspection of these plots reveals the following features: (i) in general, the secondary 16day $(s=0)$ wave amplifies when the primary SPW1 (first amplification) and 16-day W1 waves are strong, and (ii) some anti-correlation can be distinguished between the 16-day W1 wave and the 16-day $(s=0)$ wave particularly when the zonally symmetric wave amplifies (near days 80-100 for both latitudes).

Usually the growth of the secondary waves comes at the expense of some of the primary waves (or both). Therefore, the process of non-linear interaction would reduce the amplitude of this primary wave which supplies an energy feed while producing other waves. In this case apparently mainly the 16-day W1 wave supplies energy feed for the 16-day $(s=0)$ wave because only its amplitude decreases at the time when the amplitude of the secondary 16-day $(s=0)$ wave increases. Therefore, the correlation between the amplitudes of the secondary 16-day $(s=0)$ wave with those of the primary 16-day $\mathrm{W} 1$ wave will indicate if really this primary wave supplies energy for the growth of the 16-day $(s=0)$ wave. The bottom row of plots of Fig. 7 shows the time-height cross section of the cross-correlation coefficients between the amplitudes of the 16-day W1 wave and the 16-day $(s=0)$ secondary wave. The cross-correlation coefficients are calculated by using 32-day time segment (twice the period of the considered wave), which is incremented through the time series in steps of 1 day. The correlations for both latitudes are mainly negative (to facilitate the inspection, the areas with positive correlations are shaded) when the secondary 16-day $(s=0)$ wave amplifies (near days 60-100). Therefore, the secondary 16day $(s=0)$ wave grows mostly at the expense of the primary 16-day W1 wave.

We apply similar approach in studying the secondary 11day $(s=0)$ wave. In this case the second primary wave should be the eastward or westward 11-day travelling wave. The phase analysis of both 11-day waves indicated that the wave travelling eastward at low latitudes cannot propagate vertically upward above $\sim 40 \mathrm{~km}$ height, so this wave probably has no contribution to the forcing of the secondary 11day $(s=0)$ wave at tropical stratosphere. For high latitudes, most probably both, eastward and westward travelling 11day waves take part in the forcing process because both waves are vertically upward propagating waves. Figure 8 is similar to Fig. 7, but the additional middle column of plots shows the amplitudes of: the primary SPW1 (upper plot), the 11-day E1 primary wave (second plot) and the 11-day $(s=0)$ secondary wave (third plot) at $50^{\circ} \mathrm{N}$ again for the period of time November-March. An inspection of these plots reveals similar features to those shown in Fig. 7: (i) in general, the secondary 11-day $(s=0)$ wave amplifies when the primary SPW1 (both amplifications) and 11-day W1 waves (at both latitudes) and 11-day E1 wave at $50^{\circ} \mathrm{N}$ are strong, and (ii) some anti-correlation can be distinguished between the amplitudes of the secondary and the primary traveling waves, but for the high latitudes the situation is more complex.

The bottom row of plots of Fig. 8 shows: (i) the timeheight cross section of the cross-correlation coefficients between the amplitudes of the 11-day W1 wave and the 11day $(s=0)$ secondary wave at $50^{\circ} \mathrm{N}$ (left plot), (ii) the same but between the amplitudes of the 11-day E1 wave and the 11-day $(s=0)$ secondary wave at $50^{\circ} \mathrm{N}$ (middle plot), and (iii) the cross-correlation coefficients between the amplitudes of the 11-day W1 wave and the 11-day $(s=0)$ secondary wave at $25^{\circ} \mathrm{N}$ (right plot). In this case the cross-correlation 

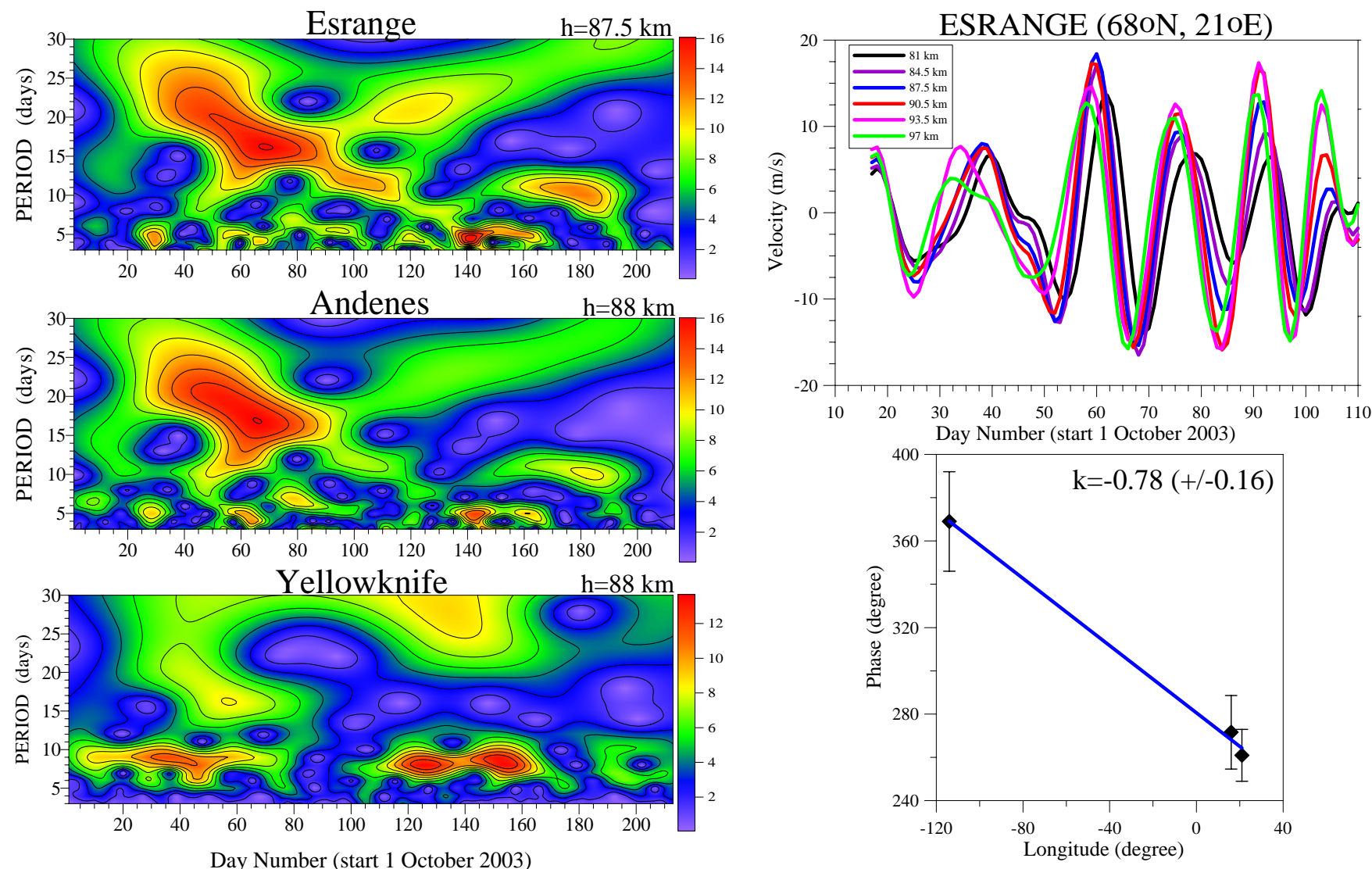

Fig. 10. Wavelet amplitude spectra (left column of plots) calculated in the period range from 3 to 30 days for the zonal wind at $\sim 88 \mathrm{~km}$ height measured at Esrange, Andenes and Yellowknife. The right column of plots shows the 16-day filtered zonal wind data at Esrange (upper plot) and the longitudinal distribution of the 16-day wave phases (bottom plot).

coefficients are calculated by using 22-day time segment (twice the period of the considered wave), which again is incremented through the time series in steps of 1 day. The correlations for low latitudes are mainly negative when the secondary 11-day $(s=0)$ wave amplifies, however this relationship is better expressed during the first (main) amplification of the 11-day $(s=0)$ wave (near days 60-100). Therefore, the secondary 11-day $(s=0)$ wave at tropical latitudes grows mostly at the expense of the primary 11-day W1 wave. For high latitudes both primary traveling waves seem to supply energy feed to the secondary 11-day $(s=0)$ wave, however, for the first (main) amplification their contribution is not simultaneous; the 11-day E1 wave has main energy contribution between days 60-80, while the 11-day W1 wave later, between days 80-110. For the second amplification of the secondary wave (days 120-160) both traveling waves supply energy feed, but the 11-day E1 wave seems to be the main supplier. The cross-correlation analysis reveals, that the 11day $(s=0)$ wave at high latitudes grows at the expense of both primary 11-day W1 and E1 waves.

\section{Latitudinal coupling in the mesosphere}

\subsection{Mean zonal wind at high- and low-latitude}

The latitudinal dynamical coupling in the MLT region during the winter of 2003/2004 has been investigated by using the zonal wind data from eleven radars: 4 at high latitudes $\left(63-69^{\circ} \mathrm{N}\right), 4$ at high-middle latitudes $\left(52-55^{\circ} \mathrm{N}\right)$ and 3 at tropical latitudes $\left(21-31^{\circ} \mathrm{N}\right)$. Table 1 lists the radar locations, the type of the instrument and the height range of available measurements. The zonal mean zonal wind UKMO data at $0.3 \mathrm{hPa}(56-58 \mathrm{~km})$ pressure level actually give some information for the base of the mesosphere. The upper plot of Fig. 9 shows the height-time cross section of the zonal mean zonal wind at pressure level $0.3 \mathrm{hPa}$ during the period of time between November 2003 and March 2004. The latitudinal relationship between high- and low latitudes is very similar to that at $1 \mathrm{hPa}$ (upper plot of Fig. 1), or the high-latitude negative perturbations are linked with positive ones at tropical latitudes before and during the onset of the SSW (near days 70-100), but later the positive perturbations observed between $50-70^{\circ} \mathrm{N}$ are associated with the negatives anomalies at low-latitudes (near days 120-160). 

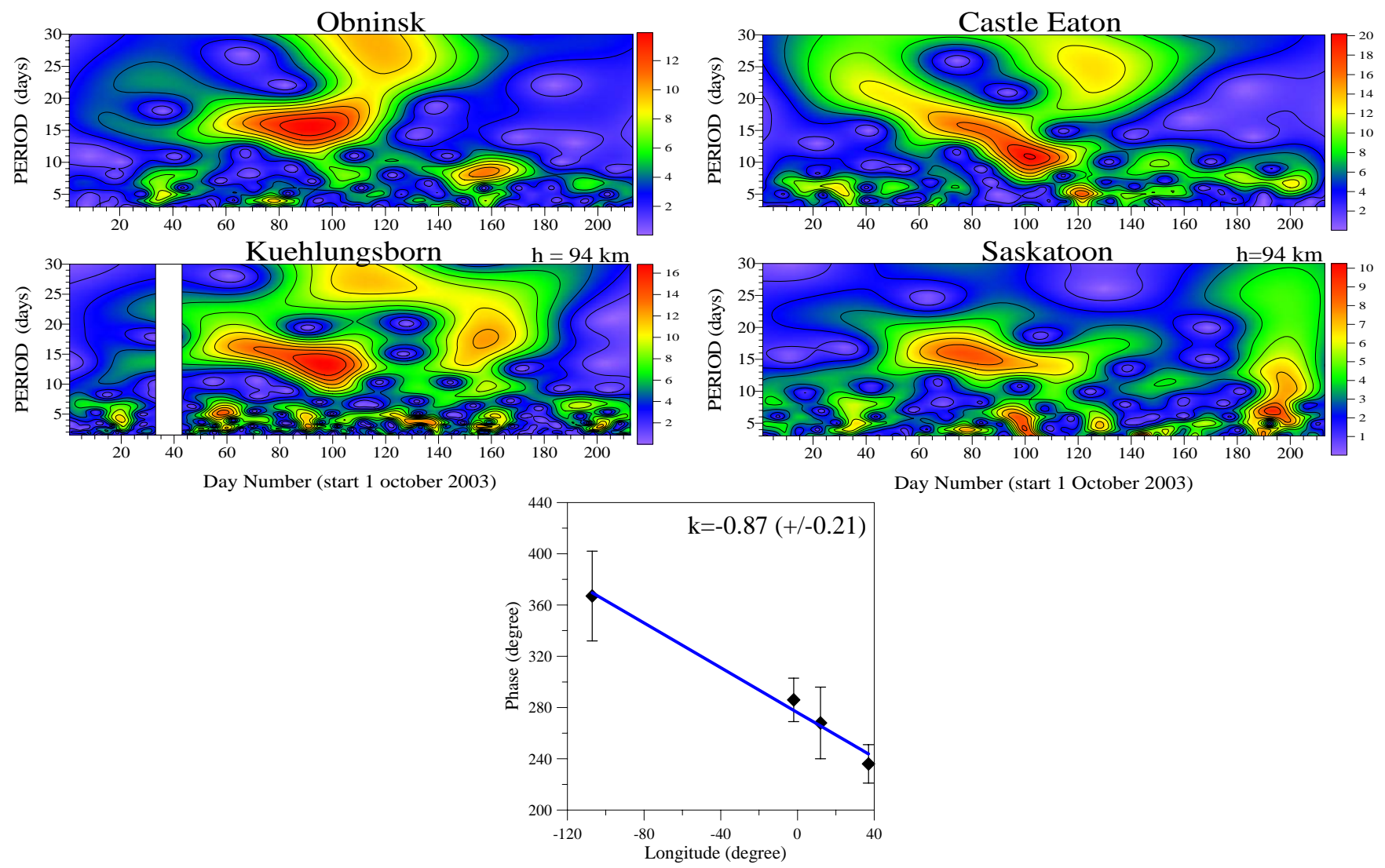

Fig. 11. Wavelet spectra calculated in the period range from 3 to 30 days for the zonal wind measured at the mid-latitude stations: Obninsk and Castle Eaton (upper row) and Kuehlungsborn and Saskatoon (bottom row). The bottom plot shows the longitudinal distribution of $\sim 16$-day wave phases.

The exact zonal mean zonal wind for the mesosphere cannot be obtained from the radar measurements because the available radars are too few in number to perform a two-dimensional analogue of the Lomb-Scargle periodogram method used for analysis of the UKMO data. We have only 4 radars situated at high latitudes, 4 radars at high-middle latitudes and 3 radars at the tropics which cover mainly half of the parallel circle. Hence their average at a height of 81$82 \mathrm{~km}$ (for high and tropical latitudes) and at $\sim 90 \mathrm{~km}$ (for high-middle and tropical latitudes) will be used as an approximation of the zonal mean zonal wind. We note that two of the radars at high-middle latitudes are without height information (Obninsk and Castle Eaton) and this prevents the calculation of the average zonal wind at $\sim 80 \mathrm{~km}$ for highmiddle latitudes. The middle plot of Fig. 9 shows a comparison between the calculated mean zonal winds at $\sim 81-82 \mathrm{~km}$ for high (black) and tropical (blue) latitudes, while the bottom plot - a comparison between the mean zonal winds at $\sim 90 \mathrm{~km}$ height for high-middle (purple) and tropical (red) latitudes (the error bars indicate the standard deviation of the mean). The following mean features can be distinguished: (i) in general, the background zonal winds at high and low latitudes have opposite relationship before and during the onset of the SSW (days 60-100); while the high-latitude mean zonal wind decelerates/reverses its direction the tropical eastward wind strengthens, and (ii) there is also a clear opposite relationship between the zonal wind perturbations with periods of planetary waves at high and low latitudes; they are well outlined before and during the onset of the SSW (days 60-100), where at both heights ( $~ 80$ and $90 \mathrm{~km})$ the negative high-latitude perturbations are associated with positive tropical disturbances. Similarly to the stratosphere, later near days $120-160$ another out-of phase perturbations can be distinguished well visible at $90 \mathrm{~km}$ height. In this case however, there is a difference between the two heights; the relationship between the perturbations at $\sim 80 \mathrm{~km}$ height near days $120-$ 160 is similar to that at stratosphere and lower mesosphere ( $0.3 \mathrm{hPa}$, upper plot of Fig. 9), or the positive disturbances at high latitudes are linked with the negative ones at low latitude, while that at $\sim 90 \mathrm{~km}$ height is the opposite, or the negative disturbances at high latitudes are linked with the positive ones at low latitude.

The result of Fig. 9 indicated that the latitudinal relationship between the dynamical regimes at high- and low-latitude 
mesosphere is very similar to that found in the stratosphere (see Fig. 1) and most probably it is produced by the zonally symmetric waves as in the stratosphere. The opposite relationship between the variability of the MLT zonal wind at high and low latitudes shown in the middle and bottom plot of Fig. 9 is an indication for the presence of such waves in the MLT zonal wind. However, there is, an important difference between the results shown in Figs. 1 and 9; while the result of Fig. 1 is based on the zonal mean zonal wind representation (where travelling and SPWs are not present) that in Fig. 9 - on a simple averaging of the zonal wind measured only by 4 (high and high-middle latitudes) or even by 3 (tropical latitudes) radars. Therefore, the results obtained in this way mean that the zonal MLT wind could be affected by the travelling and SPW waves present in the zonal wind.

Regardless of the deficiency of the mean zonal wind representation used for the mesosphere (instead of that based on the zonal mean zonal wind) the presence of the zonally symmetric waves in the MLT region could be very probable. First, we found that the zonally symmetric waves are vertically upward propagating waves, so they could penetrate into the mesosphere (we, however, cannot prove this). Second, these waves are generated by a nonlinear coupling between the SPW1 and travelling waves with zonal wavenumber 1, or particularly the $\sim 16-\mathrm{W} 1$ and 11-day W1 waves at high and low latitudes and the $\sim 11$-day E1 wave only at high latitudes. In this case, if we find the above mentioned travelling waves in the MLT zonal wind data, this could be an indirect evidence for a possible in-situ forcing of the zonally symmetric waves in the mesosphere.

We should note that from the radar wind measurements we cannot extract the SPW1. However, there are satellite observations utilized for defining the climatology of the SPWs in the MLT region (Smith, 1997; Wang et al., 2000; Forbes et al., 2002), as well as some numerical simulations (Miyahara, 1985; Pogoreltsev and Sukhanova, 1993). The observations show that the SPW1 has the largest amplitude in the mesosphere zonal wind and it occurs regularly in winter time of the NH. The presence of the SPW1 in the mesosphere (up to $80 \mathrm{~km}$ ) particularly for the period of time JanuaryMarch 2004 (when there are SABER data for high latitudes in the $\mathrm{NH}$ ) has been supported by the SABER measurements (Pancheva et al., 2008). Therefore, there is a solid base for assuming that the stratospheric amplifications of the SPW1 could be seen in the mesosphere as well (at least up to $80 \mathrm{~km})$.

The next step in our analysis is to see if there are $\sim 16$ - and $\sim 11$-day zonally travelling waves in the zonal MLT winds at high and tropical latitudes and, if they exist, to clarify their zonal structure.

\section{$4.2 \sim 16$-day wave at high- and low-latitude}

The analysis of the zonal wind data at high latitudes reveals that the strongest wave observed before the onset of the SSW
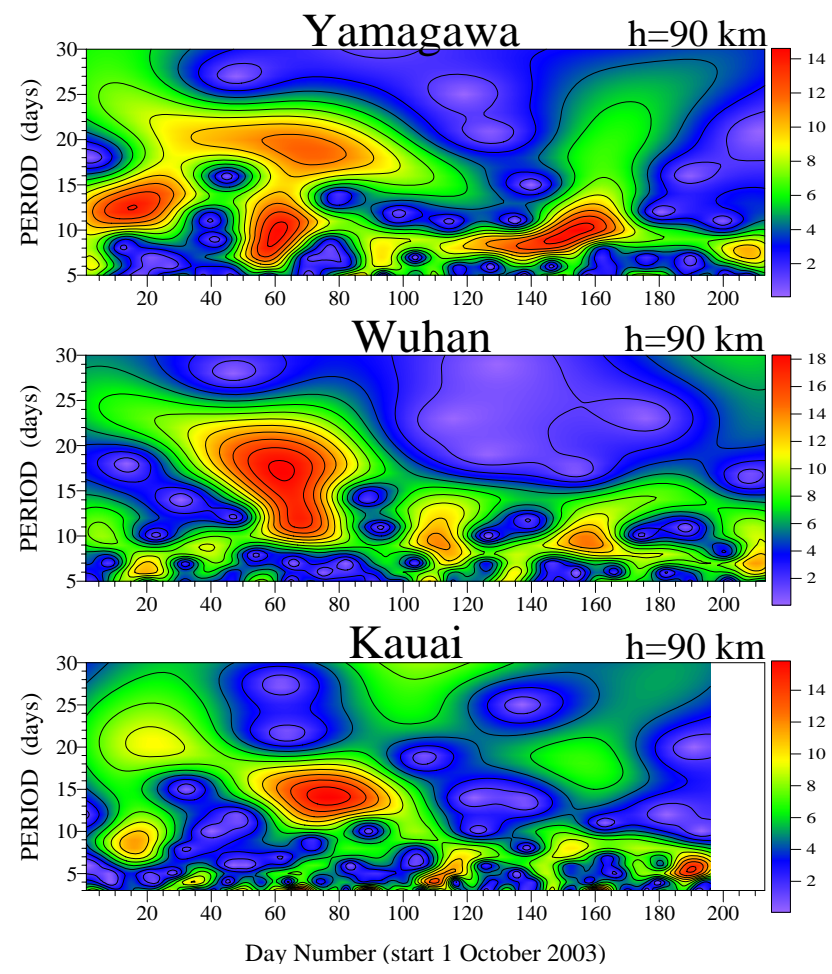

Fig. 12. Wavelet spectra calculated in the period range from 3 to 30 days for the zonal wind at $90 \mathrm{~km}$ height measured at tropical stations Yamagawa, Wuhan and Kauai.

has a period of 15-17 days. At most of the stations its amplitude maximizes near $88-93 \mathrm{~km}$ height. Figure 10 shows the wavelet amplitude spectra (left) calculated in the period range from 3 to 30 days for the zonal wind at $\sim 88 \mathrm{~km}$ height measured at 3 high-latitude stations. The data from the fourth high latitude station, Poker Flat, also hint at long-period variability before the onset of the SSW but, for altitudes higher than $84 \mathrm{~km}$, they have many gaps and the wavelet transform cannot be applied to them (it requires only short-term gaps that can be interpolated). All spectra indicate a peak near 16-17 days, which maximizes near days 60-70. The detailed analysis of this oscillation revealed that it is an upward propagating wave with vertical wavelength $\sim 50-52 \mathrm{~km}$. The upper right plot in Fig. 10 shows the 16-day filtered zonal wind data at Esrange in order to demonstrate the downward phase progression of this wave. The longitudinal distribution of the 16-17-day wave phases revealed that it is a westward propagating wave with a zonal wavenumber close to 1 (bottom right panel of Fig. 10).

Figure 11 shows the wavelet spectra calculated from the zonal wind measured at the mid-latitude stations. Recall that the meteor radars at Obninsk and Castle Eaton operate without height determination so the results represent a sampling of winds in the MLT region with weighting reflecting the vertical distribution of meteor echoes. This distribution is strongly peaked at heights near $90-95 \mathrm{~km}$. A peak 

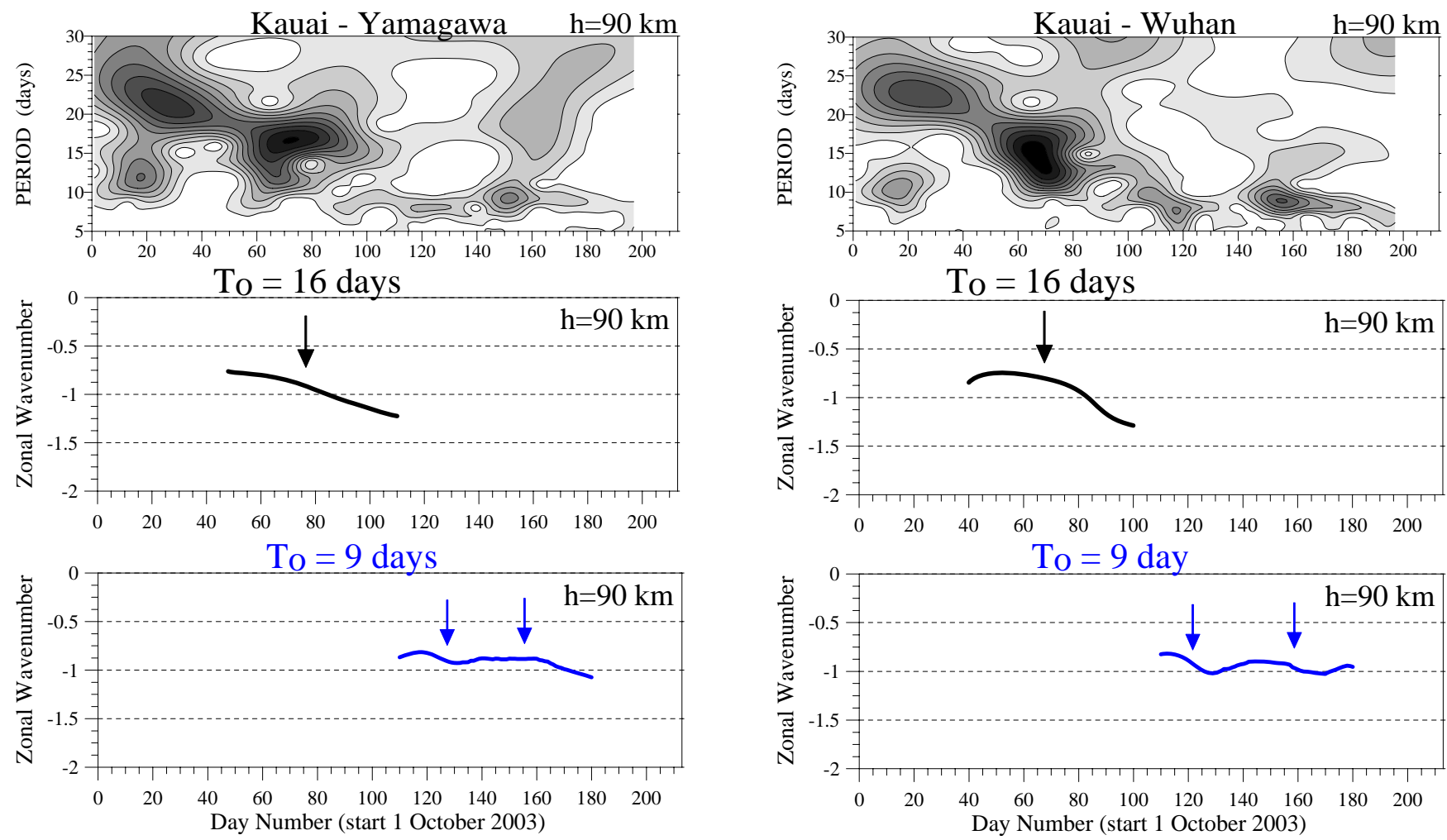

Fig. 13. Cross wavelet power spectra calculated for the couple of stations: Kauai-Yamagawa and Kauai-Wuhan (upper row of plots). The middle and bottom row of plots show the zonal wavenumbers of the 16- and 11-day waves, respectively, calculated from the wavelet phase difference between both couple of stations.

with period 15-17 days can be distinguished in all 4 stations; however the oscillations at Kuhlungsborn and Castle Eaton have periods that decrease with time from 16-17 days to $12-13$ days. The oscillation is observed before and during the onset of the SSW. The longitudinal distribution of the phases revealed that, similarly to the high latitudes, the 15-17-day oscillation is a westward propagating wave with a zonal wavenumber close to 1 (bottom plot of Fig. 11).

The analysis of the zonal wind data at low latitudes indicates also intense planetary wave activity before the onset of the SSW, but the observed periods differ from 15 at Kauai to 19-20 days at Yamagawa. Figure 12 shows the wavelet spectra calculated from the zonal wind at $90 \mathrm{~km}$ height where the wave amplitudes maximize. To estimate the zonal structure of the 15-20-day waves observed in the tropical MLT zonal wind we use cross-wavelet analysis applied to the distant couple of stations: Kauai-Yamagawa and Kauai-Wuhan. The upper row of plots in Fig. 13 shows the cross wavelet power which indicates simultaneous presence of waves with the same period at both stations. For the both couple of stations a clear peak at 16 days centred near days $60-80$ can be seen. The middle row of plots shows the zonal wavenumber of the 16-day wave calculated from the wavelet phase difference between both couple of stations and in this case it is close to -1 , or the 16-day wave propagates westward with zonal wavenumber 1 .

The above analysis clearly indicated that there is a $\sim 16$ day $\mathrm{W} 1$ wave present in the zonal wind at high- and lowlatitude mesosphere which amplifies before and during the onset of the major SSW (near days 60-100). Its amplification occurs at the time when the $\sim 16$-day $(s=0)$ wave is observed in the stratosphere and when the first out-of-phase perturbations are registered in the mean zonal wind of highand low-latitude MLT (Fig. 9).

\section{$4.3 \sim 11$-day wave at high- and low-latitude}

The analysis of the zonal wind data at high latitudes reveals that the $\sim 11$-day wave is present in the data, but it can be easily distinguished in the wavelet spectra for altitudes where the 16-day wave is not so strong; or these are altitudes higher than $90-93 \mathrm{~km}$, or lower than $80 \mathrm{~km}$. Figure 14 (left column of plots) shows the wavelet spectra for stations Esrange, Andenes and Yellowknife calculated for altitude $\sim 94 \mathrm{~km}$, while the upper plot of the right column shows the spectrum of Poker Flat calculated for $72 \mathrm{~km}$ height. All wavelet spectra show the peaks at $\sim 10$ days centred near days $80-100$ and 120-160. The longitudinal distribution of the 10-day wave 

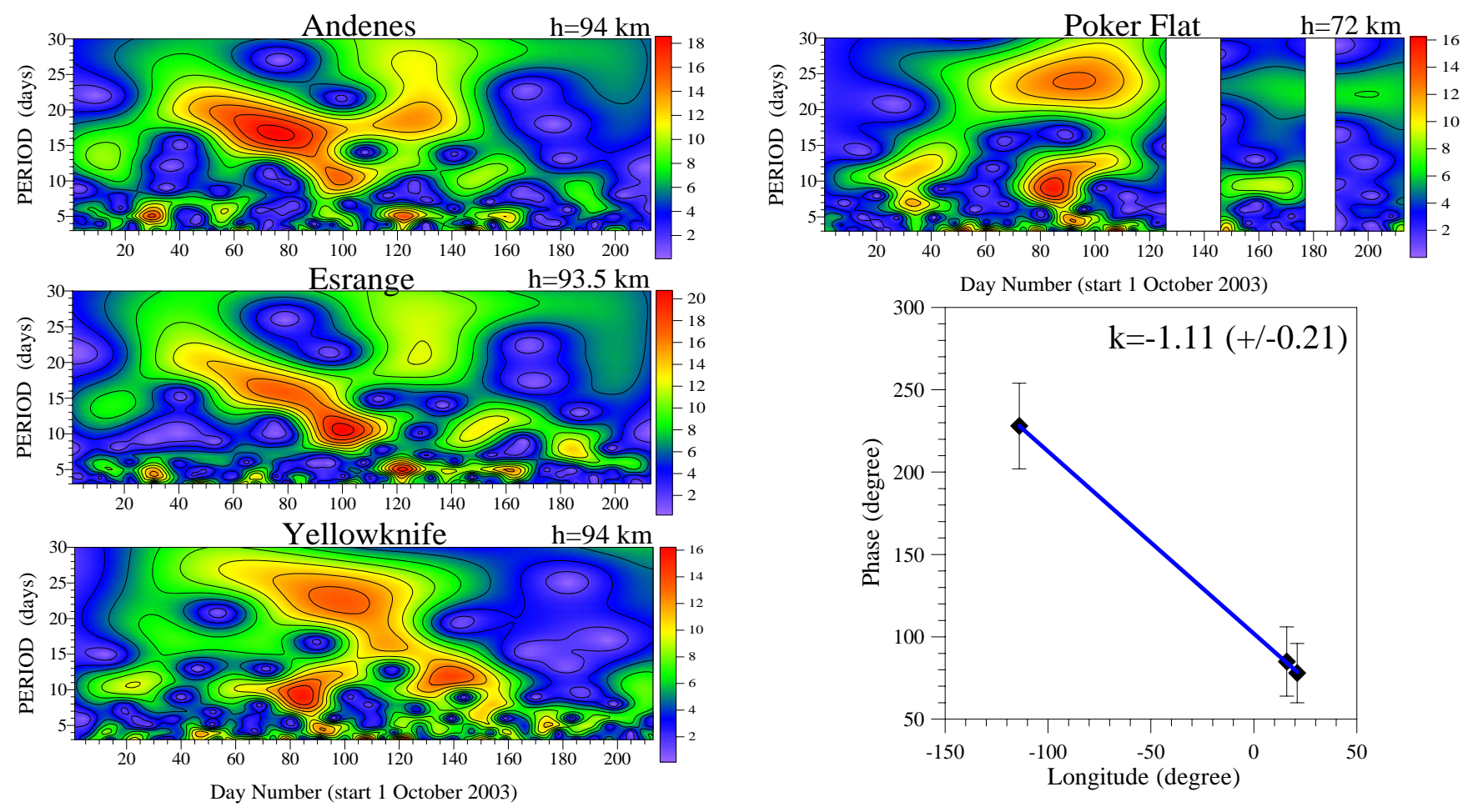

Fig. 14. Wavelet spectra calculated in the period range from 3 to 30 days for the zonal wind at $94 \mathrm{~km}$ height measured at high-latitude stations Esrange, Andenes and Yellowknife (left column of plots), while the upper right plot shows the wavelet spectra calculated for zonal wind at $72 \mathrm{~km}$ height measured at Poker Flat.

phases (for the peak centred near days 80-100) revealed that it is a westward propagating wave with a zonal wavenumber close to 1 (bottom right plot of Fig. 14).

The $\sim 11$-day wave is present in the zonal wind data of the high-middle latitude stations as well. A peak at $\sim 10$ days centred near days $140-160$ can be seen at all stations in Fig. 11, however this perturbation is too weak and we were not able to determine its longitudinal structure.

The $~ 11$-day wave is observed in the tropical MLT zonal wind as well. Clear peaks at period $8-10$ days can be seen in Fig. 12 for all tropical stations near days 100-160. The crosswavelet power shown in Fig. 13 (upper row of plots) also indicates a simultaneous presence of the $\sim 9$-day wave at all stations. The bottom row of plots shows the zonal wavenumber of the $\sim 9$-day wave calculated from the wavelet phase difference between both couple of stations and in this case, similarly to the high latitudes, it is close to -1 , or the $\sim 9$-day wave propagates westward with zonal wavenumber 1 .

The above analysis showed the presence of $\sim 11$-day W1 wave in the zonal wind at high- and low-latitude mesosphere which amplifies before and during the onset of the major SSW (near days 80-100) and later near days 120-160. Its amplification coincides with the time when the $\sim 11$-day $(s=0)$ wave is observed particularly in the upper stratosphere and when out-of-phase perturbations are registered in the mean zonal wind of high- and low-latitude MLT (Fig. 9). We recall that in the forcing of the $\sim 11$-day $(s=0)$ wave, particularly in the high-latitude stratosphere, an $\sim 11$-day E1 wave has a contribution as well. We were, however, unable to find evidence for the presence of this wave in the high-latitude MLT zonal wind. A possible reason is that the number of the radars is far from being sufficient for applying a twodimensional (time-longitude) analogue of the Lomb-Scargle periodogram method used in the UKMO data analysis which allows us to separate the waves with similar periods but different direction of propagation. As we have already mentioned some disturbances found in the MLT zonal wind could be affected by a perturbation with similar period but with different zonal structure.

\section{Summary and conclusion}

The present work is focused on the coupling of the dynamical regimes at high- and low-latitude stratosphere and mesosphere during the major SSW in the Arctic winter of $2003 / 2004$. The UKMO zonal wind data were used to explore the latitudinal coupling in the stratosphere, while the coupling in the mesosphere was investigated by neutral wind measurements from eleven radars situated at high, highmiddle and tropical latitudes. 
The main problem solved in this study is related to clarifying the type of waves producing the inverse relationship between the variability of the zonal mean flows at high- and low-latitudes related to the major SSW in the Arctic winter of 2003/2004 and defining their forcing mechanism. It was found that this opposite relationship is produced by globalscale zonally symmetric waves with periods of: $\sim 22-23$, $\sim 16$ - and $\sim 11$ days. These waves are generated by a nonlinear interaction between the SPW1 and the travelling wave with the same period and zonal wavenumber 1 . The detailed analysis of the $\sim 16$ - and $\sim 11$-day ( $s=0$ ) waves indicated that: (i) the $\sim 16$-day ( $s=0)$ wave at high and low latitudes is generated by the nonlinear interaction between the SPW1 and the $\sim 16$-day W1 wave; (ii) the $\sim 11$-day $(s=0$ ) wave at low latitudes is generated by the coupling between the SPW1 and the $\sim 11$-day $\mathrm{W} 1$ wave, while at high latitudes besides this coupling the interaction between the SPW1 and 11-day E1 wave has a contribution as well. It was also found that the zonally symmetric waves grow mostly at the expenses of the traveling waves.

It was found that the latitudinal relationship between the variability of the mean zonal wind at high- and low-latitude mesosphere is very similar to that in the stratosphere (Fig. 9) and that it could be produced by zonally symmetric waves. This result for the mesosphere, however, was obtained by a simple averaging of the zonal wind measured at different stations instead of that used in the stratosphere and based on the zonal mean zonal wind representation. Hence, it has to be accepted only as a sign of an opposite relationship between the dynamical regimes of high- and low-latitude mesosphere related to the major SSW in the Arctic winter of 2003/2004. We, however, found indirect evidence supporting the presence of zonally symmetric waves in the mesosphere. The analysis of the radar data clearly indicated the presence of $\sim 16$ - W1 and $\sim 11$-day $\mathrm{W} 1$ waves in the zonal winds at highand low-latitude mesosphere which amplify at the time when the $\sim 16$ - and $\sim 11$-day zonally symmetric waves were observed in the stratosphere. The presence of the above mentioned travelling waves in the mesosphere which originate from the stratosphere could generate in-situ the zonally symmetric waves by interacting with the SPW1. In this way, the inverse relationship observed in Fig. 9 between the variability of the mean zonal wind at high- and low-latitude mesosphere could be easily produced. It is worth mentioning also that this case-study clearly indicates the mutual relationship between the vertical and latitudinal dynamical coupling in the stratosphere-mesosphere system during the SSW events.

Acknowledgements. We are grateful to the UKMO and the BADC for the access to the data on http://www.badc.rl.ac.uk/data/assim.

Topical Editor U.-P. Hoppe thanks G. Shepherd and another anonymous referee for their help in evaluating this paper.

\section{References}

Angelats i Coll, M. and Forbes, J. M.: Nonlinear interactions in the upper atmosphere: The $s=1$ and $s=3$ nonmigrating semidiurnal tides, J. Geophys. Res., 107, A8, doi:10.1029/2001JA900179, 2002.

Cevolani, G.: Long period waves in the middle atmosphere: response of mesospheric and thermospheric winds to recent minor stratospheric warmings at mid-latitudes, Ann. Geophys., 7, 451458, 1989,

http://www.ann-geophys.net/7/451/1989/.

Cho, Y.-M., Shepherd, G. G., Won, Y.-I., Sargoytchev, S., Brown, S., and Solheim, B.: MLT cooling during stratospheric warming events, Geophys. Res. Lett., 31, L10104, doi:10.1029/2004GL019552, 2004.

Coy, L., Siskind, D. E., Eckermann, S. D., McCormack, J. P., Allen, D. R., and Hogan, T. F.: Modelling the August 2002 minor warming event, Geophys. Res. Lett., 32, L07808, doi:10.1029/2005GL022400, 2005.

Dowdy, A. J., Vincent, R. A., Murphy, D. J., Tsutsumi, M., Riggin, D. M., and Jarvis, M. J.: The large-scale dynamics of the mesosphere-lower thermosphere during the Southern Hemisphere stratospheric warming of 2002, Geophys. Res. Lett., 31, L14102, doi:10.1029/2004GL020282, 2004.

Forbes, J. M., Zhang, X., Ward, W., and Talaat, E. R.: Climatological features of mesosphere and lower thermosphere stationary planetary waves within $\pm 40^{\circ}$ latitude, J. Geophys. Res., 107 , D17, doi:10.1029/2001JD001232, 2002.

Fritz, S. and Soules, S. D.: Large-scale temperature changes in the stratosphere observed from Nimbus III, J. Atmos. Sci., 27, 10911097, 1970.

Gregory, J. B. and Manson, A. H.: Winds and wave motions to $110 \mathrm{~km}$ at mid-latitudes: III. Response of the mesospheric and thermospheric winds to major stratospheric warmings, J. Atmos. Sci., 32, 1676-1681, 1975.

Hernandez, G.: Climatology of the upper mesosphere temperature above South Pole $\left(90^{\circ} \mathrm{S}\right)$; Mesospheric cooling during 2002, Geophys. Res. Lett., 30(10), 1535, doi:10.1029/2003GL016887, 2003.

Hoffmann, P., Singer, W., and Keuer, D.: Variability of the mesospheric wind field at middle and Arctic latitudes in winter and its relation to stratospheric circulation disturbances, J. Atmos. Solar-Terr. Phys., 64, 1229-1240, 2002.

Hoffmann, P., Singer, W., Keuer, D., Hocking, W., Kunze, D., and Murayama, Y.: Latitudinal and longitudinal variability of mesospheric winds and temperatures during stratospheric warming events, J. Atmos. SolAR-Terr. Phys., 69, 2355-2366, 2007.

Jacobi, C., Kürschner, D., Muller, H. G., Pancheva, D., Mitchell, N. J., and Naujokat, B.: Response of the mesopause region dynamics to the February 2001 stratospheric warming, J. Atmos. Solar-Terr. Phys., 65, 843-855, 2003.

Julian, P. K. and Labitzke, K. B.: A study of atmospheric energetics during the January-February 1963 stratospheric warming, J. Atmos. Sci., 22, 597-610, 1965.

Liu, H.-L. and Roble, R. G.: A study of a self-generated stratospheric sudden warming and its mesospheric-lower thermospheric impacts using the coupled TIME-GCM/CCM3, J. Geophys. Res., 107(D23), 4695, doi:10.1029/2001JD001533, 2002.

Liu, H.-L. and Roble, R. G.: Dynamical Coupling of the stratosphere and mesosphere in the 2002 Southern Hemisphere major 
stratospheric sudden warming, Geophys. Res. Lett., 32, L13804, doi:10.1029/2005GL022939, 2005.

Lomb, N. R.: Least-squares frequency analysis of unequally spaced data, Astrophys. Space Sci., 39, 447-462, 1975.

Longuet-Higgins, M. S.: The eigenfunctions of Laplace's tidal equations over a sphere, Philos. Trans. R. Soc. London, Ser. A, 262, 511-607, 1968.

Lysenko, I. A., Portnyagin, Y. I., Greisiger, K. M., and Sprenger, K.: Some peculiarities of the atmospheric circulation at the altitude of 90-100 km over Europe in winter 1972-73, Zeitschrift für Meteorology, 25, 213-217, 1975.

Manney, G. L., Krüger, K., Sabutis, J. L., Sena, S. A., and Pawson, S.: The remarkable 2003-2004 winter and other recent warm winters in the Arctic stratosphere since the late 1990s, J. Geophys. Res., 110, D04107, doi:10.1029/2004JD005367, 2005.

Manson, A. H. and Meek, C. E.: Climatologies of mean winds and tides observed by medium frequency radars at Troms $\varnothing\left(70^{\circ} \mathrm{N}\right)$ and Saskatoon $\left(52^{\circ} \mathrm{N}\right)$ during 1987-1989, Can. J. Phys., 69, 966-975, 1991.

Matsuno, T.: A dynamical model of the stratospheric sudden warming, J. Atmos. Sci., 28, 1479-1494, 1971.

Miyahara, S.: Suppression of stationary planetary waves by internal gravity waves in the mesosphere, J. Atmos. Sci., 42, 100-107, 1985.

Mukhtarov, P., Pancheva, D., Andonov, B., Mitchell, N. J., Merzlyakov, E., Singer, W., Hocking, W., Meek, C., Manson, A., and Murayama Y.: Large-scale thermodynamics of the stratosphere and mesosphere during the major stratospheric warming in 2003/2004, J. Atmos. Sol.-Terr. Phys., 69, 2338-2354, 2007.

Murayama, Y., Igarashi, K., Rice, D., Watkins, B., Collins, R., Mizutani, K., Saito, Y., and Kainuma, S.: Medium frequency radars in Japan and Alaska for upper atmosphere observations, IEICE Trans., E83-B, 1996-2003, 2000.

Myrabø, H. K., Deehr, C. S., and Lybekk, B.: Polar cap OH airglow rotational temperatures at the mesopause during a stratospheric warming event, Planet. Space Sci., 32, 853-856, 1984.

Pancheva, D. and Mukhtarov, P.: Wavelet analysis on transient behaviour of tidal amplitude fluctuations observed by meteor radar in the lower thermosphere above Bulgaria, Ann. Geophys., 18, 316-331, 2000, http://www.ann-geophys.net/18/316/2000/.

Pancheva, D., Mukhtarov, P., and Andonov, B.: Zonally symmetric oscillations in the Northern hemisphere stratosphere during the winter of 2003/2004, Geophys. Res. Lett., 34, L04807, doi:10.1029/2006GL028666, 2007.

Pancheva, D., Mukhtarov, P., Mitchell, N. J., Merzlyakov, E., Smith, A. K., Andonov, B., Singer, W., Hocking, W., Meek, C., Manson, A., and Murayama, Y.: Planetary Wave Coupling of the Stratosphere and Mesosphere during the Major Stratospheric Warming in 2003/2004, J. Geophys. Res.-Atmos., 113, in press, 2008.
Pogoreltsev, A. I. and Sukhanova, S. A.: Simulation of the global structure of stationary planetary waves in the mesosphere and lower thermosphere, J. Atmos. Sol.-Terr. Phys., 55, 33-40, 1993.

Riggin, D. M., Lieberman, R. S., Vincent, R. A., Manson, A. H., Meek, C. E., Nakamura, T., Tsuda, T., and Portnyagin, Yu. I.: The two-day wave during the boreal summer of 1994, J. Geophys. Res., 108, D08110, doi:10.1029/2003JD004493, 2004.

Scargle, J. D.: Studies in astronomical time series analysis. II. Statistical aspects of spectral analysis of unevenly spaced data, Astrophys. J., 263, 835-853, 1982.

Shepherd, M. G., Wu, D. L., Fedulina, I. N., Gurubaran, S., Russell, J. M., Mlynczak, M. G., and Shepherd, G.: Stratospheric warming effects on the tropical mesospheric temperature field, J. Atmos. Solar-Terr. Phys., 69, 2309-2337, 2007.

Singer, W., Hoffmann, P., Keuer, D., Schminder, R., and Kürschner, D.: Wind in the middle atmosphere with partial reflection measurements during winter and spring in middle Europe, Adv. Space Res., 12(10), 299-302, 1992.

Siskind, D. E., Coy, L., and Espy, P.: Observations of stratospheric warmings and mesospheric coolings by the TIMED SABER instrument, Geophys. Res. Lett., 32, L09804, doi:10.1029/2005GL022399, 2005.

Smith, A. K.: Stationary planetary waves in upper mesospheric winds, J. Atmos. Sci., 54, 2129-2145, 1997.

Swinbank, R. and O'Neill, A.: A stratosphere-troposphere data assimilation system, Mon. Weather Rev., 122, 686-702, 1994.

Swinbank, R. and Ortland, D. A.: Compilation of the wind data for the Upper Atmosphere Research Satellite (UARS) Reference Atmosphere Project, J. Geophys. Res., 108(D19), 4615, doi:10.1029/2002JD003135, 2003.

Walterscheid, R. L., Sivjee, G. G., and Roble, R. G.: Mesospheric and lower thermospheric manifestations of a stratospheric warming event over Eureka, Canada $\left(80^{\circ} \mathrm{N}\right)$, Geophys. Res. Lett., 27(18), 2897-2900, 2000.

Wang, D. Y., Ward, W. E., Shepherd, G. G., and Wu, D. L.: Stationary planetary waves inferred from WINDII wind data taken within altitudes 90-120 km during 1991-1996, J. Atmos. Sci., 57, 1906-1918, 2000.

Whiteway, J. A. and Carswell, A. I.: Rayleigh lidar observations of thermal structure and gravity wave activity in the high arctic during a stratospheric warming, J. Atmos. Sci., 51, 3122-3136, 1994.

Xiong, J., Wan, W., Ning, B., and Liu, L.: First results of the tidal structure in the MLT revealed by Wuhan Meteor Radar $\left(30^{\circ} \mathrm{N}\right.$, 114.5 E), J. Atmos. Solar-Terr. Phys., 66, 675-682, 2004. 